\title{
The informational content of prices when policy makers react to financial markets
}

\author{
Christoph Siemroth* \\ University of Essex
}

September 27, 2018

\begin{abstract}
When can policy makers use policy-relevant information from financial market prices and how does policy affect price informativeness? I analyze a novel setting with noise where a policy maker tries to infer information about a state variable from prices to improve policy decisions, and policy in turn affects asset values. I derive a necessary and sufficient condition for the possibility of information revelation in equilibrium, which might not be possible if the policy reaction to prices punishes traders for revealing their information. If the policy maker is uninformed, then policy objectives do not change price informativeness, but they do if the policy maker has independent information about the state. I also analyze policy maker transparency, and find that policy makers with objectives having a large impact on asset values should publish their information before trading to make prices more informative. In other cases, intransparency can be optimal.
\end{abstract}

Keywords: Asymmetric Information, Central Bank Transparency, Financial Markets, Policy Risk, Price Informativeness, Rational Expectations Equilibrium

JEL Classification: D53, D82, D84, G10

*University of Essex, Department of Economics, Wivenhoe Park, Colchester, CO4 3SQ, UK. E-mail: christoph.siemroth@essex.ac.uk. 


\section{Introduction}

One of the key insights in financial economics is that asset prices can reveal the information that traders have. Besides traditional financial markets that can aggregate information about the state of companies or the economy as a whole, prediction markets have been used successfully to forecast non-financial outcomes such as elections (e.g., Berg et al., 2008), fulfilling project deadlines (e.g., Cowgill and Zitzewitz, 2015), or whether scientific studies will be successfully replicated (e.g., Dreber et al., 2015). Thus, financial market prices can potentially be valuable tools in helping policy makers by providing information or forecasts in many domains. For example, regulators might learn about bank health from bond prices and could use it for intervention decisions. And central banks already monitor asset prices to learn about economic fundamentals or inflation expectations and use it to improve policy (e.g., Bernanke and Woodford, 1997).

However, if policy affects asset values, then as soon as policy reacts to asset prices, it can change the incentives of traders and affect the informativeness of prices. Most of the above results were arguably observed in settings where traders did not anticipate that a policy maker would react to (the information contained in) the asset prices. So an important question is if and when traders would still trade in a way that reveals (some of) their information if they correctly anticipate that this information is used for policy purposes.

This paper answers this question by deriving the conditions (in terms of policy maker preferences and asset properties) under which the policy maker can and cannot learn from prices to improve policy in equilibrium in a setting with noise. These results can be useful to design institutions/assets that allow for better information revelation. Moreover, the paper investigates how policy maker preferences/objectives affect how much information is revealed by prices. Finally, I use the model to address the question of policy maker transparency, i.e., under which conditions a policy maker should reveal her private information to other market participants if the goal is to extract information from the market.

To answer these questions, I adapt a CARA-normal asset pricing model with noise to include a policy maker, who moves after and potentially reacts to the financial market. The value of the risky asset depends both on a fundamental state variable $\theta$ (which is standard) and a policy variable $i$ (new). Informed traders obtain signals about the state $\theta$, so financial market prices can potentially reveal some information about the state. The optimal policy of the policy maker depends on the state $\theta$, and since the policy maker is not perfectly informed about the state $\theta$, she tries to infer information about the state from financial market prices and in effect policy reacts to these prices.

A potential problem in similar settings without noise - where prices react to policy and policy reacts to prices - is that traders may not reveal their information and there may not even exist an equilibrium (e.g., Bernanke and Woodford, 1997; Bond et al., 2010). This is because the policy reaction to prices can punish traders for revealing their information, 
so that the pricing problem is a self-defeating prophecy (Siemroth, 2017). To give one informal example of a self-defeating prophecy, consider a bank bond whose value depends on the financial health of the bank (the state $\theta$ ) and a regulator decision (policy decision $i$ ). Informed traders know something about bank health that the regulator does not know. If bank health is bad, traders can trade at low prices, but this reveals the need for an intervention to the regulator, ${ }^{1}$ who for example gives a guarantee to the bank which improves the asset value. Thus, the trader forecast of a low asset value is falsified by the regulator decision and traders lose money since they sold below value. If traders, on the other hand, trade at high prices despite low bank health, then it signals that there is no need for an intervention, hence there is no intervention and the asset value remains low. Again, traders lose money since they bought above value. Forward looking traders anticipate these adverse policy reactions and therefore no equilibrium may exist. This problem can occur because asset values are endogenous, so that the action of an agent may punish traders by affecting asset values. To the best of my knowledge, this is the first paper to investigate the problem of self-defeating prophecies in a noisy rational expectations equilibrium (REE) framework.

Adapting a new solution approach for noisy REE (e.g., Breon-Drish, 2015), I can solve for equilibria with an uninformed policy maker who has general preferences allowing for non-linear policy reaction functions, which would be hard to deal with in the standard solution approach. I derive a sufficient and necessary condition for the existence of (partially) revealing equilibria in a fairly general class of equilibria that includes and generalizes the usual linear noisy REE, which answers the question under which circumstances market prices can help policy makers.

This condition requires invertibility of a risk-weighted expectation of the asset value in a noisy statistic of the state, which converges to the asset value evaluated at the optimal policy in the noiseless limit. ${ }^{2}$ Thus, the condition ensures that the endogenous asset valueafter taking into account the policy reaction - really is strictly monotone in the information that the price reveals, otherwise traders would be better off not clearing the market. Put differently, the condition implicitly defines the set of policy maker preferences for which the policy reaction to prices does not punish traders for revealing information. In terms of price informativeness, if the policy maker is uninformed, then the policy maker objectives/policy do not affect how much information is revealed in equilibrium, only whether a (partially revealing) equilibrium exists.

In the online appendix, I also demonstrate that noise can solve the problem of self-

\footnotetext{
${ }^{1}$ Clearly, the regulator expectations about how the state maps into prices is endogenous in equilibrium. In this example, the equilibrium candidate is that traders trade at low prices for bad bank health and trade at high prices for good bank health, which generates a revealing price function and the expectations described. A self-defeating prophecy is a setting where no revealing price function is an equilibrium.

${ }^{2}$ Thus, in the limit, the condition is the same as in models without noise (Siemroth, 2017), which might justify using these simpler models in environments with "little noise." This result can be useful for applied theory work since the noisy setting imposes some functional form limitations that are typically not a problem in models without noise, so the latter can analyze a broader set of applications and policies.
} 
defeating prophecies in some settings such as the one considered by Bernanke and Woodford (1997): Because noise prevents full revelation of trader information, the informed retain incentives to trade on information, making prices informative while they would not be without noise. Thus, paradoxically, noise might sometimes improve information revelation in settings where asset values are endogenous to policy.

If the policy maker is informed, i.e., receives imperfect private signals about the state, then policy maker preferences not only determine whether informative equilibria exist, but also how informative they are. Because asset values are affected by policy decisions, a policy maker with independent information introduces additional 'policy risk' in asset returns beyond the usual risk over asset fundamentals, since traders do not know the independent information of the policy maker (which in part determines policy). This is why policy maker preferences influence how aggressively the informed trade on information. Moreover, policy risk can induce strategic complementarity leading to multiple equilibria, while the setting with uninformed policy maker features unique equilibria.

Comparative statics show that more extreme policy maker preferences (in the sense that policies with a large influence on asset values are preferred) tend to decrease price informativeness, because the policy reaction adds risk by amplifying the asset value variance and due to the uncertainty underlying the policy reaction. ${ }^{3}$ Consequently, the model suggests that market-based policy — which uses market information as input - works better with policies that have a smaller impact on asset values. The comparative statics also show that policies which move against fundamental shocks can increase information revelation by markets, because policy dampens the asset variance from the perspective of the traders. Since the policy maker can make better decisions and achieve a higher utility with better information, a larger price informativeness coincides with better real decisions and welfare in this setting. Thus, the model enables an analysis of the impact of information contained in financial market prices on real decisions and welfare.

If the policy maker has private information, then a natural question is under which conditions this information should be revealed publicly before trading, which is especially important in the context of central bank transparency. The model shows that the policy maker should reveal her information if she has extreme intervention preferences. This is because transparency removes the policy risk for traders, while intransparency implies a lot of policy risk for traders and therefore limits trading on information, making prices less informative. Hence, transparency is to the policy maker's benefit especially when policy has a large impact on the market. However, there are cases where intransparency (not revealing the private information) is optimal, for example if the policy maker has mild preferences such that policy moves against fundamental shocks. In this case, policy acts like a dampening force against asset value movements driven by fundamentals. Intransparency has a positive

\footnotetext{
${ }^{3}$ Hence, the model shows that some policy objectives interacting with fundamentals can result in excess volatility, whereas existing explanations for this phenomenon rely on behavioral factors (e.g., Daniel et al., 1998) or other feedback effects (e.g., Ozdenoren and Yuan, 2008).
} 
effect here, as it ensures that fundamentals and policy are two negatively correlated shocks from the perspective of traders, which reduces the asset value variance.

This paper is related to the theoretical literature investigating the self-defeating prophecy or "double endogeneity" problem, including Woodford (1994) and Bernanke and Woodford (1997) on central banks, Birchler and Facchinetti (2007) on banking supervision with a good description of the formal problem, Bond et al. (2010) on corrective actions, and Siemroth (2017) unifying some of the earlier contributions and adding applications such as corporate prediction markets. In all of these models, a decision maker reacts to information revealed by the financial market and in turn affects asset values, which can lead to equilibrium nonexistence. Moreover, Prescott (2012) and Sundaresan and Wang (2015) study a related problem in contingent capital models, where a mechanical rule instead of a decision maker reacts to financial market prices, which can also cause a self-defeating prophecy problem. None of these models feature a setting with noise, which is crucial to investigate how much information is revealed, since equilibrium prices in the absence of noise are typically fully revealing (e.g., Radner, 1979). This paper adds to the existing knowledge by showing that self-defeating prophecies can occur also in settings with noise, but also that noise can solve the self-defeating prophecy problem in some cases.

Bond and Goldstein (2015) (BG in short) analyze a related CARA-normal noisy REE model with policy maker, but in their setting the asset value does not directly depend on $\theta$, only indirectly via the government action that depends on the state $\theta$. Hence, in their model, traders use their information to predict the government's information and action rather than the state. For this reason, their implications for transparency are different, because as soon as the government's information is public in their setting, there is no need for traders to trade on their information, so price informativeness suffers. In the setup here, the conclusions on transparency are more nuanced, and transparency typically helps the policy maker to obtain more information from the market, while there are some cases where transparency is undesirable. Moreover, the setup in BG does not allow for self-defeating prophecies, so the present paper contributes by analyzing if and when these can arise, which makes a major qualitative difference when equilibrium non-existence is interpreted as breakdown of information revelation. This is especially true in my case of an uninformed policy maker with non-linear policy rules, which allow for a larger set of applications.

$\mathrm{BG}$ also investigate when it pays for the government to commit itself to using more or less financial market information to conduct policy (relative to the ex post optimal rule). While commitment is not the focus here, my analysis contributes by parameterizing the informed policy maker objectives and deriving the effect of preference parameters on price informativeness, thereby answering how policy maker objectives affect the financial market. Moreover, I contribute by discussing the possibility and causes of equilibrium multiplicity due to the presence of the policy maker.

The current paper is also related to the literature investigating the real effects of financial 
markets via an informational channel. In most of this literature, the 'real effect' is the financial market information impact on corporate decisions, as in Goldstein and Guembel (2008); Foucault and Gehrig (2008); Ozdenoren and Yuan (2008); Goldstein et al. (2013); Edmans et al. (2015); Dow et al. (2017). Typically, these papers rule out equilibrium non-existence due to feedback via technical assumptions, while I highlight that these cases can be important even from an applied point of view. In the present paper, the real effect includes any effect of price information on third parties, which depending on the application can include central bank policy and their consequences, banking regulation, or corporate decisions.

\section{The model}

\section{$2.1 \quad$ Setup}

In this section, I extend a standard constant absolute risk aversion (CARA)-normal model by introducing a policy maker. The equilibrium market clearing price $p$ is affected by the realization of a random noise variable $u$, which is independent of the state $\theta$. In the common interpretation, $u$ is the aggregate net demand of noise traders, whose trading activity (due to exogenous reasons such as liquidity shocks) is independent of the price/state.

For rational traders and the policy maker, the noise shocks introduce a difficulty in extracting information from the price: A high asset price may indicate favorable information about the fundamental $\theta$, or it may indicate a lot of noise trader purchases $u$ which are unrelated to fundamentals. Consequently, traders and the policy maker will only be able to make stochastic inferences about the realization of $\theta$ from the market price, and cannot perfectly infer $\theta$ from the market price as in a model without noise (Siemroth, 2017).

In the definition of the environment I heavily borrow notation from Vives (2010), who provides a detailed derivation of the standard linear noisy rational expectations equilibrium without policy maker.

Assets. The financial market consists of a riskless asset (return normalized to zero) and a risky asset. The value/payoff of the risky asset is an additively separable function of the fundamental $\theta$ and policy choice $i$,

$$
A(\theta, i)=\theta+f(i)
$$

with arbitrary function $f: I \rightarrow \mathbb{R}$. I will later have to impose more restrictions on function $f$ if the policy maker receives a signal. In one possible application, the asset is a bank stock, $\theta$ is the bank health, and $i$ is a regulator action (such as requiring more costly reserves, giving a government guarantee, etc.) that affects the bank stock value. The regulator action might 
depend on bank health, say because the government does not want to give costly guarantees unless bank health is very poor.

State variable and information. All random variables in the model are normally distributed. ${ }^{4}$ The fundamental or state variable is $\theta$ with common prior distribution $\mathcal{N}\left(\bar{\theta}, 1 / \tau_{\theta}\right)$. Private trader signals about $\theta$ are denoted $s_{j}=\theta+\varepsilon_{j}, \varepsilon_{j} \sim \mathcal{N}\left(0,1 / \tau_{\varepsilon}\right)$, and the private policy maker signal is $s_{p}=\theta+\varepsilon_{p}, \varepsilon_{p} \sim \mathcal{N}\left(0,1 / \tau_{\varepsilon}\right)$ (in case of an informed policy maker, otherwise no private signal). Finally, aggregate noise trader net demand is denoted by $u \sim \mathcal{N}\left(0,1 / \tau_{u}\right)$. All variances are positive and finite, i.e., $0<\tau_{\theta}, \tau_{\varepsilon}, \tau_{u}<\infty$, and the realizations of the noise variables $\varepsilon_{p}, \varepsilon_{j}, u$ are independent of $\theta$. The distributions of all random variables (but not their realizations) are common knowledge.

Traders. There is a continuum of traders $j \in[0,1]$, and all $j$ have a CARA utility function defined over investment returns $\pi_{j}$,

$$
U_{j}\left(\pi_{j}\right)=-\exp \left(-\rho_{j} \pi_{j}\right) \text { with } \pi_{j}=(\theta+f(i)-p) x_{j}
$$

and net demand $x_{j}$ for the risky asset. A share $\mu \in(0,1]$ of traders are informed, receive conditionally independent signals $s_{j}$ about the realization of $\theta$, have a coefficient of absolute risk aversion $\rho_{I}>0$, and use demand strategies $X_{I}\left(p, s_{j}\right): \mathbb{R}^{2} \rightarrow \mathbb{R}$ to be specified later. A share $(1-\mu) \in[0,1)$ of traders are uninformed, have a coefficient of absolute risk aversion $\rho_{U}>0$, and use demand strategies $X_{U}(p): \mathbb{R} \rightarrow \mathbb{R}$ to be specified later. CARA-utility functions exhibit no wealth effects, hence I normalize wealth to zero without loss of generality. As is standard, traders have no budget constraints in this model; demands are bounded by the degree of risk aversion.

Policy maker. The policy maker ("she" throughout the paper) has a utility function defined over state $\theta$ and policy $i, U(\theta, i),{ }^{5}$ for which $i(z)=\arg \max _{i} \mathbb{E}_{\theta}[U(\theta, i) \mid z]$ exists for any normally distributed signal $z$ about the state $\theta$, and is unique and continuous in $z$. Uniqueness will be used to establish equilibrium uniqueness, but if we did not insist on a unique equilibrium, then it could be relaxed. For non-triviality, I assume the optimal policy $i(z)$ depends on information $z$, otherwise the policy maker has no need to infer information from financial market prices. I will distinguish the cases of an uninformed policy maker, who only knows the prior distribution of $\theta$, and an informed policy maker, who also receives an imperfect signal $s_{p}$ about the state $\theta$. The model considers settings where traders know something about $\theta$ that the policy maker would like to know for policy purposes, and the policy maker can try to infer information about $\theta$ from financial market prices, which may

\footnotetext{
${ }^{4}$ While Breon-Drish (2015) has shown that normality of random variables can sometimes be generalized to random variables from the exponential family in these types of models (possibly at the cost of closed form solutions), I will stick to normal random variables in this paper and keep the focus on the novel aspect of the policy maker affecting asset values.

${ }^{5}$ This formulation does not allow the policy maker to derive utility from trading. While this is an interesting extension that might add incentives to use policy to manipulate asset values in order to increase trading profits, the focus here is on learning information from prices rather than manipulating the market, so this question is left for future research.
} 


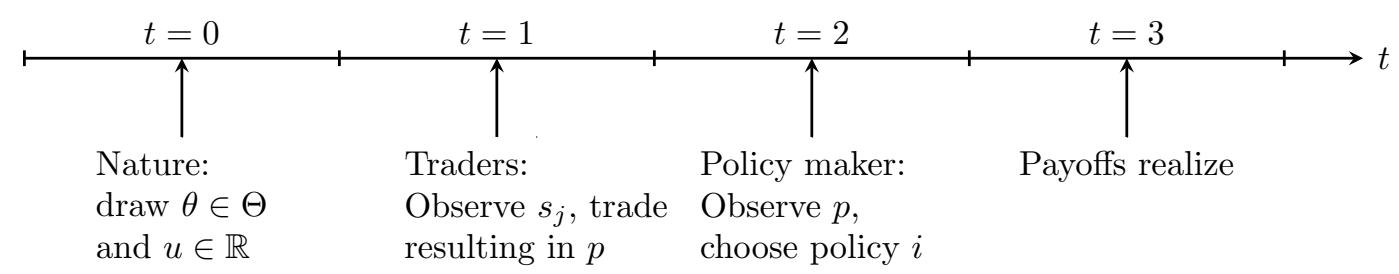

Figure 1: Timeline with traders and policy maker.

reveal some of the trader information (depending on the equilibrium). The label "policy maker" suggests that the decision maker is a public sector official, but she may be any decision maker such as a firm executive whose decision affects asset values.

The timing is as depicted in Figure 1: First, nature draws all random variables including state $\theta$, then trading leads to a market clearing price $p$, the policy maker sets $i$, and finally payoffs realize. Since the policy maker can condition on the price, but traders cannot condition on the policy, the model yields the same equilibria if we assume simultaneous decisions of traders and policy maker.

This model nests the standard CARA-normal rational expectations model, which is the special case $f(i)=0$, i.e., where the policy maker does not affect asset values. This CARAnormal parametrization facilitates a comparison with the existing literature, which heavily relies on this framework since Grossman and Stiglitz (1980) and Hellwig (1980). Indeed, Vives (2010) calls it the workhorse model in the study of financial markets with asymmetric information.

A noisy rational expectations equilibrium with an informed policy maker is defined as follows (the definition for an uninformed policy maker is easily adapted).

Definition 1. A noisy rational expectations equilibrium is a set of trading strategies contingent on the price and available information, $X_{I}\left(p, s_{j}\right)$ for all $j \in[0, \mu]$ and $X_{U}(p)$ for all $j \in(\mu, 1]$, an optimal policy function $i\left(p, s_{p}\right)$, and a measurable price functional $P(\theta, u)$ such that

1. the market for the risky asset clears:

$$
\int_{0}^{\mu} X_{I}\left(p=P(\theta, u), s_{j}\right) d j+\int_{\mu}^{1} X_{U}(p=P(\theta, u)) d j+u=0 \text { a.s. }
$$

2. all traders $j$ use optimal demand strategies given the available information,

$$
\begin{gathered}
X_{I}\left(p, s_{j}\right) \in \arg \max _{x} \mathbb{E}_{\theta, s_{p}}\left[U_{j}\left(\left(\theta+f\left(i\left(p, s_{p}\right)\right)-p\right) x\right) \mid p=P(\theta, u), s_{j}\right] \forall j \in[0, \mu], \\
X_{U}(p) \in \arg \max _{x} \mathbb{E}_{\theta, s_{p}}\left[U_{j}\left(\left(\theta+f\left(i\left(p, s_{p}\right)-p\right)\right) x\right) \mid p=P(\theta, u)\right] \forall j \in(\mu, 1],
\end{gathered}
$$


3. the policy maker sets an optimal policy given the available information,

$$
i\left(p, s_{p}\right) \in \arg \max _{i} \mathbb{E}_{\theta}\left[U(\theta, i) \mid p=P(\theta, u), s_{p}\right]
$$

\subsection{Results with an uninformed policy maker}

I begin the analysis with a policy maker who does not receive an independent signal on the fundamental $\theta$, hence the only information available to her (apart from the prior) is contained in market prices. Usually, the CARA-normal setup is used for its tractability and simple closed-form solutions. However, the typical guess-and-verify approach to solving the model, guessing a linear equilibrium price function, can only analyze cases with linear policy reaction functions. For nonlinear reaction functions, the approach requires knowledge of the functional form of the equilibrium price function. Thus, the approach is unsuitable to derive a condition for equilibrium existence depending on policy maker preferences. Breon-Drish (2015) recently demonstrated another solution approach in a model without policy maker.

Adapting this new approach in an extended model with policy maker, I can solve for equilibria with nonlinear policy reaction functions if the policy maker is uninformed, and can derive a condition for equilibrium existence depending on policy maker preferences. The only other noisy model of policy maker-trader interaction that I am aware of (Bond and Goldstein, 2015) only considers linear policy functions. Since the equilibria can be solved in closed-form, the model is useful for many applications. The prime application is monetary policy: There is considerable evidence that central bank policy reacts nonlinearly or asymmetrically to changes in price levels and stock prices (e.g., Weise, 1999; Kim et al., 2005; Surico, 2007; Ravn, 2012).

\subsubsection{Equilibrium}

With an uninformed policy maker, I will consider the class of equilibria where demand functions of the informed traders are possibly nonlinear in the price but additively separable from signal $s_{j}$,

$$
X_{I}\left(s_{j}, p\right)=a s_{j}-g_{I}(p), X_{U}(p)=-g_{U}(p),
$$

and where the equilibrium price function $P(\theta, u)$ is continuous. ${ }^{6}$ This class includes and generalizes the linear equilibria solved for in the standard guess-and-verify approach. Equilibrium existence or non-existence is understood within this equilibrium class.

Definition 2 (Quasi-linear equilibrium). The class of equilibria where the net demand

\footnotetext{
${ }^{6}$ While equilibria in the class of continuous equilibria are unique (see Breon-Drish, 2015 and Proposition 1 below), Pálvölgyi and Venter (2015) show for the classical Grossman and Stiglitz (1980)-model that a continuum of discontinuous equilibria with varying price informativeness exists besides the standard linear equilibrium. Thus, we may view the focus on continuous price functions as a reasonable equilibrium refinement.
} 
function of the informed traders takes the form $X_{I}\left(s_{j}, p\right)=a s_{j}-g_{I}(p)$ for constant a and function $g_{I}: \mathbb{R} \rightarrow \mathbb{R}$, with continuous price function $p=P(\theta, u)$, is called quasi-linear.

The main question is under which conditions a (partially) revealing equilibrium exists, i.e., in which situations the policy maker can learn from financial market prices and use the information for policy purposes.

In the following I outline how to derive this condition. The main steps are to determine the informational content of the equilibrium price function assuming an equilibrium exists, then the policy reaction function, then the trader strategies, then the price function, and finally the condition that ensures that an equilibrium indeed exists as conjectured.

Suppose a quasi-linear equilibrium exists. Recall that the equilibrium price function $P(\theta, u)$ maps all possible realizations $(\theta, u)$ into a price $p \in \mathbb{R}$. Since we are in equilibrium, the market clearing condition is fulfilled. Using the form of the demand functions from definition 2, the market clearing condition becomes

$$
\begin{aligned}
\int_{0}^{\mu} a s_{j}-g_{I}(P(\theta, u)) d j-(1-\mu) g_{U}(P(\theta, u))+u & =0 \\
\Longleftrightarrow \frac{1}{\mu a}\left(\mu g_{I}(P(\theta, u))+(1-\mu) g_{U}(P(\theta, u))\right) & =\theta+u /(\mu a) .
\end{aligned}
$$

Only the right hand side of $(2)$ directly depends on $(\theta, u)$. Since the market clearing condition has to hold for all realizations $(\theta, u)$, it implies that the left hand side has to react to any change in $\theta+u /(\mu a)$. And since the left hand side depends on $\theta$ and $u$ only via the equilibrium price function $P(\theta, u)$, it further implies that any equilibrium price function must change with the linear statistic $z:=\theta+u /(\mu a)$, which is a noisy signal of the state $\theta$. Consequently, the price function must be invertible in $z$ and thus reveals it, so I shall write $P(z)$ instead of $P(\theta, u)$ in the following. Thus, if there is an equilibrium, then the policy maker can infer the realization $z$ from the price.

Continuity of the price function ensures that prices reveal the realization $z$ and no more, as later shown in the proof. The contribution of Breon-Drish (2015) and others is to recognize that this approach allows to pin down the information set of the uninformed (which in this section includes the policy maker) without knowing the functional form of the equilibrium price function $P(\theta, u)$. This is extremely valuable here, since the asset value (and hence asset price function) depends on the policy maker preferences and is therefore not in general linear.

The policy maker reaction function to the financial market prices is

$$
i(p) \in \arg \max _{i} \mathbb{E}_{\theta}[U(\theta, i) \mid p=P(z)]
$$

and since prices reveal the statistic $z$, I shall abuse notation and also write $i(z)$. Note that 
the policy reaction to prices is not a price effect in the classical sense, but a reaction to the information contained therein.

The asset value $\theta+f(i(p))$ conditional on the price is normally distributed ${ }^{7}$, even if the policy reaction function $i(p)$ is nonlinear, since the reaction is deterministic given $p$. For a normally distributed asset value, the demand functions of the informed traders with CARA utility are

$$
\begin{aligned}
X_{I}\left(s_{j}, p\right) & =\frac{\mathbb{E}\left[\theta+f(i(p))-p \mid s_{j}, p\right]}{\rho_{I} \operatorname{Var}\left(\theta+f(i(p))-p \mid s_{j}, p\right)}=\frac{\mathbb{E}\left[\theta \mid s_{j}, p\right]+f(i(p))-p}{\rho_{I} \operatorname{Var}\left(\theta \mid s_{j}, p\right)} \\
& =\frac{\tau_{\theta}+\tau_{\varepsilon}+(\mu a)^{2} \tau_{u}}{\rho_{I}}\left(\frac{\tau_{\theta} \bar{\theta}+\tau_{\varepsilon} s_{j}+(\mu a)^{2} \tau_{u} P^{-1}(p)}{\tau_{\theta}+\tau_{\varepsilon}+(\mu a)^{2} \tau_{u}}+f(i(p))-p\right),
\end{aligned}
$$

which is of the form $X_{I}\left(s_{j}, p\right)=a s_{j}-g_{I}(p)$ as assumed, with

$$
g_{I}(p)=-\left[\tau_{\theta} \bar{\theta}+(\mu a)^{2} \tau_{u} P^{-1}(p)+(f(i(p))-p)\left(\tau_{\theta}+\tau_{\varepsilon}+(\mu a)^{2} \tau_{u}\right)\right] / \rho_{I},
$$

and, matching the coefficient, $a=\tau_{\varepsilon} / \rho_{I}$. The demand functions of the uninformed traders are similarly

$$
X_{U}(p)=\frac{\mathbb{E}[\theta \mid p]+f(i(p))-p}{\rho_{U} \operatorname{Var}(\theta \mid p)}=\left[\tau_{\theta} \bar{\theta}+(\mu a)^{2} \tau_{u} P^{-1}(p)+(f(i(p))-p)\left(\tau_{\theta}+(\mu a)^{2} \tau_{u}\right)\right] / \rho_{U}
$$

with

$$
g_{U}(p)=-\left[\tau_{\theta} \bar{\theta}+(\mu a)^{2} \tau_{u} P^{-1}(p)+(f(i(p))-p)\left(\tau_{\theta}+(\mu a)^{2} \tau_{u}\right)\right] / \rho_{U} .
$$

Substituting $g_{I}(p), g_{U}(p)$ into the market clearing condition (2) and setting $p=P(z)$,

$$
\begin{array}{r}
-\frac{1}{\mu a}\left(\mu\left[\tau_{\theta} \bar{\theta}+(\mu a)^{2} \tau_{u} P^{-1}(P(z))+(f(i(P(z)))-P(z))\left(\tau_{\theta}+\tau_{\varepsilon}+(\mu a)^{2} \tau_{u}\right)\right] / \rho_{I}\right. \\
\left.+(1-\mu)\left[\tau_{\theta} \bar{\theta}+(\mu a)^{2} \tau_{u} P^{-1}(P(z))+(f(i(P(z)))-P(z))\left(\tau_{\theta}+(\mu a)^{2} \tau_{u}\right)\right] / \rho_{U}\right)=z .
\end{array}
$$

As established before, market clearing requires the equilibrium price function $P(z)$ to be invertible. Thus, we can rewrite $P^{-1}(P(z))=z$ and, abusing notation, $i(P(z))=i(z)$ with $i(z)=\arg \max _{i} \mathbb{E}[U(\theta, i) \mid P(z)]=\arg \max _{i} \mathbb{E}[U(\theta, i) \mid z]$. These are the price terms in $(7)$ that only depend on the information contained in prices and not on the actual price to be paid for the asset. The remaining price terms are the price to be paid, and so we can rearrange

\footnotetext{
${ }^{7}$ Recall that the equilibrium price function reveals the normally distributed signal $z$, thus the posterior distribution over $\theta$ is normal as well.
} 
the market clearing condition (7) to obtain the equilibrium price function,

$$
\begin{array}{r}
P(z)=\left\{z\left(1+\mu^{2} a \tau_{u} / \rho_{I}+(1-\mu) \mu a \tau_{u} / \rho_{U}\right)+\mu\left[\tau_{\theta} \bar{\theta}+f(i(z))\left(\tau_{\theta}+\tau_{\varepsilon}+(\mu a)^{2} \tau_{u}\right)\right] /\left(\rho_{I} \mu a\right)\right. \\
\left.+(1-\mu)\left[\tau_{\theta} \bar{\theta}+f(i(z))\left(\tau_{\theta}+(\mu a)^{2} \tau_{u}\right)\right] /\left(\rho_{U} \mu a\right)\right\} \\
/\left\{\mu\left(\tau_{\theta}+\tau_{\varepsilon}+(\mu a)^{2} \tau_{u}\right) /\left(\rho_{I} \mu a\right)+(1-\mu)\left(\tau_{\theta}+(\mu a)^{2} \tau_{u}\right) /\left(\rho_{U} \mu a\right)\right\} .
\end{array}
$$

This is an explicit expression for the price function given optimal demands and policy if the price function reveals $z$, and if traders anticipate the policy reaction $i(z)$. Notice that the price function is unique if $i(z)$ is unique. For equilibrium, we still have to confirm that the price function is in fact invertible in $z$, as required for market clearing. After simplifying, $P(z)$ is invertible if and only if

$$
\begin{array}{r}
z\left[1+\mu^{2} a \tau_{u} / \rho_{I}+(1-\mu) \mu a \tau_{u} / \rho_{U}\right]+f(i(z))\left[\mu\left(\tau_{\theta}+\tau_{\varepsilon}+(\mu a)^{2} \tau_{u}\right) /\left(\rho_{I} \mu a\right)\right. \\
\left.+(1-\mu)\left(\tau_{\theta}+(\mu a)^{2} \tau_{u}\right) /\left(\rho_{U} \mu a\right)\right]
\end{array}
$$

is invertible in $z$. These terms capture how aggregate net demand for the asset changes if $z$ changes. But (9) might not be invertible if $f(i(z))$ is at least locally decreasing, i.e., if the policy reaction reduces the asset value sufficiently for a larger realization of $z$. Thus, the only possible cause of non-invertibility and consequently equilibrium non-existence is the policy reaction function $i(z)$ to information $z$ : In the standard CARA-normal case without policy maker, which is the special case $f(i(z))=0$ for all $z$, the equilibrium price function is linearly increasing and an equilibrium always exists. But in the more general case with policy maker an equilibrium does not have to exist, nor does the equilibrium price function have to be increasing if an equilibrium does exist; it depends on the policy maker preferences and hence reaction function $i(z)$ affecting the asset value.

Notice the similarity of this condition to the invertibility condition in the model without noise (Siemroth, 2017), which requires that the asset value given optimal policy and combined trader information is invertible. If we let the noise variance go to zero here $\left(\tau_{u} \rightarrow \infty\right)$, then (8) reduces to invertibility of $z+f(i(z)$ ) (i.e., the expected asset value given $z$ ), and since $z=\theta+u /(\mu a)=\theta$ a.s. for $\tau_{u} \rightarrow \infty, \theta+f(i(\theta))$ is the asset value given optimal policy and combined trader information - the same condition as in the model without noise.

As in the model without noise (Siemroth, 2017), the equilibrium non-existence result can be interpreted as a self-defeating prophecy. The price function is like a market prediction of the future asset value - the higher the trader prediction of the future asset value, the higher the prices at which they are willing to trade. And this prediction (the price function) reveals some of the trader information about the state $\theta$ to the policy maker (more precisely, it reveals the noisy statistic $z$ ). But because the policy maker reacts to this information, and 
her policy reaction in turn affects asset values, it may be that any price function (prediction) that reveals $z$ triggers a policy response that falsifies the prediction, i.e., the pricing problem is a self-defeating prophecy. Note that the problem is not the learning from prices by the policy maker per se, but rather what she does with the information and what its impact on asset values is, as shown by the $i(z)$ term in (9).

The following proposition gives the formal result.

Proposition 1. An equilibrium in the class of quasi-linear equilibria exists if and only if, for $a=\tau_{\varepsilon} / \rho_{I}$, the term (9) is invertible in $z$.

If the equilibrium exists, then it is unique in the class of quasi-linear equilibria and market prices fully reveal the noisy signal $z=\theta+u /(\mu a)$. The trader equilibrium strategies are $X_{I}\left(s_{j}, p\right)=a s_{j}-g_{I}(p)$ and $X_{U}(p)=-g_{U}(p)$ with $g_{I}(p)$ given in (4) and $g_{U}(p)$ given in (6). The equilibrium price function $P(\theta, u)=P(z)$ is given in (8).

Proof. See Appendix 3.

Interestingly, market prices are informative if an equilibrium exists, but the trading aggressiveness $a$ of the informed traders and therefore price informativeness is constant and unaffected by policy maker preferences. To illustrate Proposition 1, consider the following example.

Example 1. Consider a bank/insurance bailout scenario motivated by the AIG situation in the financial crisis of 2008, where the US government injected hundreds of billions of dollars into the business to keep it alive, since several major investment banks had acquired default insurance for their assets from AIG (see, e.g., Reuters, 2008), arguably making a bankruptcy a systemic risk. The state of the insurer/bank $\theta$ is uncertain from the perspective of the regulator, but traders have information about it and potentially reveal it by trading.

More specifically, suppose the asset value depends on the bank's cash flow, which is simply $A(\theta, i)=\theta+i$, where $\theta$ is the state of the firm/economy and $i$ is a possible cash injection by the regulator. Suppose further the regulator wants to inject cash $i>0$ if necessary to reduce the bankruptcy probability given the available information to $\alpha$, say $1 \%,{ }^{8}$ and otherwise not inject cash $(i=0)$. This is captured formally by the following regulator utility function $(\mathbf{1}\{x\}$ is the indicator function)

$$
U_{1}(\theta, i)=(\mathbf{1}\{\theta+i<T\}-\alpha)^{2} \text { with } i \geq 0
$$

where $T \in \mathbb{R}$ is a cash threshold below which the bank cannot meet its obligations and goes bankrupt. Also assume that $\tau_{\theta}=0$, i.e., the prior variance is infinite (improper prior), so that the prior is completely uninformative. Thus, only the information from the market determines the regulator's estimate of state $\theta$. In this case condition (9) simplifies to $z+f(i)$.

\footnotetext{
${ }^{8}$ Recall that in a world with normally distributed state and imperfect information, the regulator cannot rule out bankruptcy, only make it very unlikely.
} 
Can the regulator learn from the financial market if a cash injection is necessary and how large it would need to be? Suppose there is an equilibrium where the financial market price function of the asset reveals information about $\theta$ to the regulator; as established in Proposition 1, this information is the normally distributed signal $z=\theta+u /(\mu a)$. The reaction function of the regulator to this information,

$$
i_{1}(z)=\arg \max _{i \geq 0}(\operatorname{Pr}(\theta+i<T \mid z)-\alpha)^{2}
$$

is plotted in Figure 2a. For large realizations of $z$, the condition of the bank is good and the probability of default absent intervention is below $\alpha$, so the regulator does not intervene. However, for small enough $z$, the probability of bankruptcy would exceed $\alpha$ without cash injection, so the regulator sets $i>0$ such that $H(T-i \mid z)=\alpha$, where $H$ is the cdf of the posterior distribution of $\theta$ given $z$, which is normal. Because the market information $z$ is the only information about the state, $i_{1}(z)$ has a slope of -1 in this range, so that the regulator reaction "undoes" any adverse movement of the state (lower $z$ ) by a corresponding increase in cash $(i>0)$, thus keeping the bankruptcy probability given her information constant.

Now it is rather easy to check whether information revelation by the market is an equilibrium: After computing the reaction function $i_{1}(z)$, we have to confirm that condition (9) is invertible. This term is plotted in Figure 2b. Clearly, the term is constant in the range where the regulator perfectly cancels out any movement in $z$. Thus, no partially revealing equilibrium exists. This is a self-defeating prophecy: Traders, by revealing $z$, trigger a regulator reaction that affects asset values in such a way that traders do not want to reveal $z$. Hence, traders do not clear any price function that would reveal $z$.

Overall, there are policy maker utility functions for which an equilibrium exists for all parameter values, for some parameter values, or for none. Regulator preferences $U_{1}$ do not generally preclude information revelation. Indeed, this happens if and only if $\tau_{\theta}=0$, otherwise the informative prior would make the regulator more cautious in reacting to $z$. But with the condition derived in this section it is easy to check if and when an equilibrium exists for a specific utility function.

As the example illustrates, the problem of determining whether information is revealed in equilibrium is simplified considerably by making use of Proposition 1 . The problem boils down to the following cook-book approach: First, computing the policy reaction function $i(z)$, followed by checking whether $(9)$ based on $i(z)$ is invertible.

To summarize, Proposition 1 shows that noise cannot generally solve the problem of self-defeating prophecies, which has previously only been documented in models without noise (e.g., Bernanke and Woodford, 1997; Siemroth, 2017). But the online appendix also demonstrates that noise can solve the non-existence problem in certain cases. One such case is asset $a(\theta, i)=\theta+i$ and policy preferences with a reaction function $i(p)=\alpha-\mathbb{E}[\theta \mid p]$. In the noiseless limit, conjecturing that a revealing equilibrium exists, this reaction function would 


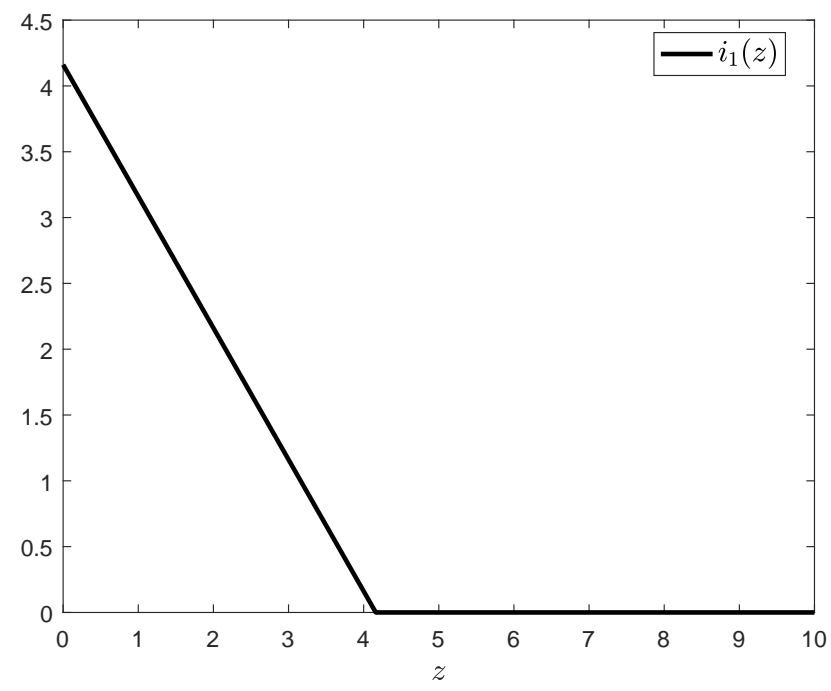

(a) Regulator reaction function

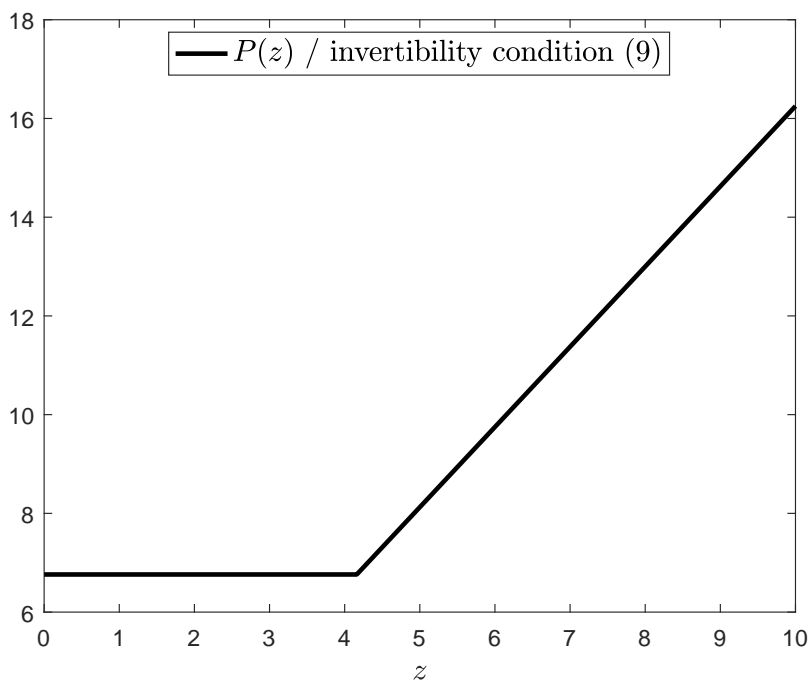

(b) Test of equilibrium invertibility condition passes

Figure 2: Policy maker reaction function and test of equilibrium condition for policy objectives $U_{1}$.

lead to a constant asset value $a(\theta, i(p))=\alpha$, hence the conjecture is wrong and no such equilibrium exists. However, for any $\tau_{u}<\infty$, a revealing equilibrium does exist, because the noise prevents that the policy maker perfectly reacts to the state, so the informed can still make profits by trading on their information, which makes the equilibrium partially revealing.

\subsubsection{Comparative statics: Price informativeness and welfare}

In this section, I derive the comparative statics regarding price informativeness if asset values are affected by the policy reaction. I argue below that price informativeness is also a 
measure of welfare for all (unmodelled) agents affected by the policy maker decision, since the policy maker decision is better the more information she has about the state. Hence, this section answers the question how financial market variables affect welfare among the agents affected by the policy decision (e.g., in the non-financial sectors).

To define a measure of price informativeness, consider the mean squared error of the policy maker "estimator" of state $\theta$ given the price signal $p$,

$$
\operatorname{MSE}(\mathbb{E}[\theta \mid p]):=\mathbb{E}\left[(\theta-\mathbb{E}[\theta \mid p])^{2} \mid p\right]
$$

which is a well known measure of the deviation or forecast error of an estimator from the variable to be estimated. To measure price informativeness, take the difference of the mean squared error of the policy maker estimate without the information contained in the market price and the mean squared error of the estimate with the information contained in the market price. Thus, the measure directly captures the differences in "forecast errors" of $\theta$ due to access to the financial market price and the information contained therein: The larger the measure, the more the price information helps to estimate $\theta$. For an uninformed policy maker, the price informativeness measure is

$$
\begin{aligned}
\mathrm{PI}_{\text {uninformed }} & :=\operatorname{MSE}(\mathbb{E}[\theta])-\operatorname{MSE}(\mathbb{E}[\theta \mid p])=\mathbb{E}\left[(\theta-\mathbb{E}[\theta])^{2}\right]-\mathbb{E}\left[(\theta-\mathbb{E}[\theta \mid p])^{2} \mid p\right] \\
& =\operatorname{Var}(\theta)-\operatorname{Var}(\theta \mid p)=\frac{1}{\tau_{\theta}}-\frac{1}{\tau_{\theta}+(\mu a)^{2} \tau_{u}}=\frac{1}{\tau_{\theta}^{2} /\left((\mu a)^{2} \tau_{u}\right)+\tau_{\theta}} .
\end{aligned}
$$

$\mathrm{PI}_{\text {uninformed }}$ is not just price informativeness for the (uninformed) policy maker, but all uninformed traders or outsiders. The following proposition is straightforward and establishes the comparative statics for price informativeness.

Proposition 2 (Comparative statics price informativeness). In the quasi-linear noisy REE with uninformed policy maker,

- larger trader signal precision $\tau_{\varepsilon}$, share of informed traders $\mu$, or noise precision $\tau_{u}$, and

- smaller prior distribution precision $\tau_{\theta}$, or risk aversion $\rho_{I}$,

increase price informativeness (10), all else equal.

Proof. See Appendix 3.

These comparative statics are the same as in a CARA-normal model without policy maker, because as established in Proposition 1, the policy reaction does not affect how aggressively the informed trade on their information (captured by demand function coefficient $\left.a=\tau_{\varepsilon} / \rho_{I}\right)$, which is the only equilibrium object in the price informativeness measure.

A central question of this paper is to what degree information from financial markets can help policy makers to improve real decisions. A welfare measure of interest is how much the 
financial market information improves the utility of the policy maker by improving decisions. We can think of the policy maker utility as a welfare measure for all the (unmodeled) nontrader individuals that are affected by the policy maker decision. ${ }^{9}$ For example, if the financial market can reveal information about future inflation shocks to the central bank, then the central bank will be better able to hit the target inflation rate or maintain price stability, thus improving welfare.

In this context, the only obstacle for the policy maker to achieve the maximum utility is that she does not perfectly know the state and hence chooses a policy that is not optimal given the state. Hence, more price informativeness improves policy decisions and thus ex ante policy maker utility. The price informativeness measure is therefore a good measure of the welfare/policy maker utility gains from the financial market information in this context.

\subsection{Results with an informed policy maker}

\subsubsection{Preliminaries}

The case of independent private information about state $\theta$ for the policy maker is not just a technical extension. This section shows that it makes policy-relevant economic difference. In particular, this section shows that independent information for the policy maker leads to policy objectives affecting the informational content of prices. In other words, depending on what the policy maker intends to do with the information from financial market prices, she may get more or less information from markets. This is not the case if the policy maker has no independent private information (see previous section). Hence, when setting policy goals, one has to keep in mind that equilibrium effects can lead the financial market to provide less information. I apply the model later in section 2.3.4 to analyze one particular policy question that recently has received a lot of attention in the context of central banking: When should a policy maker reveal his private information about $\theta$ to the market and when should it be kept confidential?

Before moving to the results, I need to cover a few technical preliminaries. If the policy maker is (imperfectly) informed, i.e., receives an imperfect signal $s_{p}=\theta+\varepsilon_{p}, \varepsilon_{p} \sim \mathcal{N}\left(0,1 / \tau_{\varepsilon}\right)$ from the same distribution as the informed trader signals, ${ }^{10}$ then the demand functions of the informed traders are not generally of the quasi-linear form as in definition 2, unless the policy reaction function is linear. This is because the independent signal $s_{p}$ allows the policy maker to react to the state $\theta$ independently of the price, so any non-linear policy reaction is a non-linear reaction to the price as well as the state. For this reason, one cannot explicitly solve for an equilibrium with the above tools if the utility function implies non-linear reactions.

\footnotetext{
${ }^{9}$ Clearly, using policy maker utility as welfare measure assumes a benevolent policy maker.

${ }^{10}$ It is easy to allow for more or less precise signals for the policy maker compared to traders, but this does not add much more insight so I avoid this complication except in the comparative statics.
} 
Consequently, throughout this section, I assume the policy maker has the quadratic utility function

$$
U(\theta, i)=\psi_{1}+\psi_{2} i-\psi_{3} i^{2} / 2+\psi_{4} i \cdot \theta+\psi_{5} \theta
$$

which implies a linear reaction function of the form

$$
i\left(p, s_{p}\right)=\beta_{1}+\beta_{2} p+\beta_{3} s_{p}
$$

where the $\beta$-coefficients are functions of the primitives. This policy maker utility function is one flexible parameterization that yields a linear reaction function, although it is not the only one. I choose it here to be able to analyze the effect of policy maker preferences on price informativeness explicitly. The coefficient on the interaction term $\psi_{4} \neq 0$ ensures that optimal policy changes with the state. The ratio of two parameters, $\psi_{4} / \psi_{3}$, will be of particular interest in this section, which determines how strongly and in which direction the optimal policy reacts to the realization of $\theta$. To see this, suppose the realization $\theta$ were known to the policy maker, then her policy reaction function would be $i(\theta)=\left(\psi_{2}+\psi_{4} \theta\right) / \psi_{3}$, so a positive $\psi_{4} / \psi_{3}$ leads to larger $i$ the larger $\theta$, and a negative $\psi_{4} / \psi_{3}$ leads to smaller $i$ with larger $\theta$. Hence, $\psi_{4} / \psi_{3}$ is the part of the utility function that affects policy and thereby asset values.

Traders' demand functions still take the quasi-linear form from definition 2, but due the the linear policy reaction function, the trader demand functions will in fact turn out to be linear in equilibrium. For later reference, trader equilibrium strategies are of the form

$$
X_{I}\left(p, s_{j}\right)=a s_{j}-c_{I} p+b_{I} \text { and } X_{U}(p)=-c_{U} p+b_{U}
$$

for informed and uninformed traders, respectively.

Finally, I need to restrict the asset value function (1) to $f(i)=i$ in order to keep the expected asset value linear in the state $\theta$, so that the asset value given state $\theta$ and policy $i$ in this section is

$$
A(\theta, i)=\theta+i
$$

\subsubsection{Equilibrium}

Although the stronger functional form assumptions on policy maker utility and asset value (compared to the previous section) guarantee that the equilibrium price function is linear and the price signal is normally distributed, which allows us to use the usual solution techniques, the equilibrium cannot be explicitly solved. This is because one equilibrium condition is a fifth degree polynomial, which does not generally admit an analytical solution. This equilibrium condition determining the equilibrium object $a$ (coefficient in demand function 
$(13))$ is $^{11}$

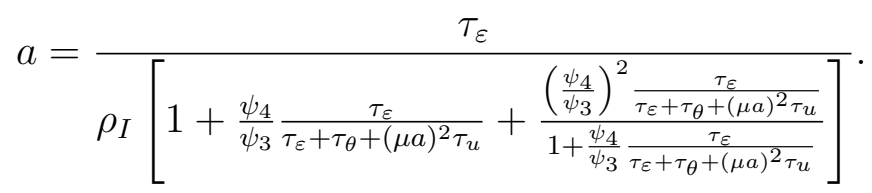

This equilibrium condition requires that the trading aggressiveness $a$, i.e., how much informed traders change their positions depending on their signals $s_{j}$, equals the change of the expected risk-weighted asset value in $s_{j}$ (this is the entire right hand side). The standard model without policy maker is the special case $\psi_{4} / \psi_{3}=0$, where $a=\tau_{\varepsilon} / \rho_{I}$ is the explicit solution that is well known from the standard CARA-normal models. For the more general case $\psi_{4} / \psi_{3} \neq 0$ with policy maker, however, condition (14) shows that trading aggressivenessand hence price informativeness - is affected by policy objectives represented by the ratio of utility function parameters $\psi_{4} / \psi_{3}$. This is because asset values are endogenous: The policy objective affects the actual policy and thereby asset values, so traders take policy objectives into account in their trading strategy.

Without solving the condition explicitly, I first establish existence of equilibrium for almost all parameter profiles, but there need not be a unique equilibrium. Then I give conditions for equilibrium uniqueness and derive comparative statics in these unique equilibria. Finally, I explain how "policy risk" affects equilibrium trading behavior and price informativeness, and how policy risk can create a form of strategic complementarity and induce multiple equilibria.

Proposition 3 (Equilibrium existence). If the policy maker is informed, then a noisy REE with linear demand and policy strategies (13) and (12), and linear price function, exists for almost all parameter values.

Proof. See Appendix 3.

In addition to the price function, a symmetric equilibrium has to determine the coefficients $\left\{a, c_{I}, b_{I}\right\}$ in the demand functions of the informed traders (13), the coefficients $\left\{c_{U}, b_{U}\right\}$ in the demand functions of the uninformed traders (13), and the coefficients $\left\{\beta_{1}, \beta_{2}, \beta_{3}\right\}$ in the reaction function of the policy maker (12).

The proof of Proposition 3 shows that there may be different equilibrium values of $a$, but all other strategy coefficients $c_{I}, c_{U}, b_{I}, b_{U}, \beta_{1}, \beta_{2}, \beta_{3}$ are unique given $a$. Thus, coefficient $a$ - how aggressively the informed trade on information - is the only source of equilibrium multiplicity. An equilibrium may fail to exist for a negligible set of parameter values because of a self-defeating prophecy, where - if prices were informative - the policy reaction to prices $i\left(p, s_{p}\right)$ would exactly cancel out any favorable information about $\theta$, so traders would have no reason to change their positions for different prices, which means that the informative

\footnotetext{
${ }^{11}$ For details on the derivation, see the proofs in appendix 3 .
} 
prices could not clear the market. This case is essentially the noisy version of the equilibrium non-existence problem in the model without noise in Bernanke and Woodford (1997).

The intuition for the equilibrium non-existence is very much as in the case of an uninformed policy maker (see especially section 2.2.1) or as in the noiseless case (Siemroth, 2017). Due to the restriction to linear reaction functions in this section, self-defeating prophecies are only a knife-edge case in $\psi_{4} / \psi_{3} \in \mathbb{R}$ space, hence an equilibrium exists for almost all parameter values. This is different in the case of an uniformed policy maker where the setting allows for non-linear reaction functions, for which equilibrium non-existence is not a knife-edge case (see Proposition 1).

Without solving for the equilibrium trading aggressiveness $a$ explicitly, it is obvious from the equilibrium condition (14) that $a=0$, i.e., a completely uninformative equilibrium, can never be a solution. Thus, noisy REE in the presence of a policy maker are always partially revealing. Moreover, if $\psi_{4} / \psi_{3}$ is not too negative, i.e., if the policy maker does not want to reduce the asset value too much with larger $\theta$, then in equilibrium informed traders will buy more of the asset the larger their signal $s_{j}$ (i.e., $a>0$ ), so the equilibrium price function is increasing in the state. This changes if $\psi_{4} / \psi_{3}$ is sufficiently negative, see the discussion of Proposition 5 below. Overall, policy maker preferences determine how much information is revealed by market prices in equilibrium, and it may be more or less than in a standard REE model without policy maker depending on $\psi_{4} / \psi_{3} \cdot{ }^{12}$

\section{Corollary 4.}

i. In any equilibrium, $a \neq 0$, i.e., market prices are informative.

ii. If $\frac{\psi_{4}}{\psi_{3}}>-\frac{\tau_{\varepsilon}+\tau_{\theta}}{\tau_{\varepsilon}}$, then $a>0$ in any equilibrium.

Proof. See Appendix 3.

Although existence of an equilibrium is generic (Proposition 3) due to the linearity of the policy maker reaction function, uniqueness (within the class of quasi-linear equilibria) is not guaranteed. The next result therefore derives simple sufficient conditions for uniqueness. Not only are the predictions of the model sharper with a unique equilibrium, it also makes comparative statics unambiguous while they are typically not if multiple equilibria exist (see next section).

The proposition shows that sufficiently strong policy maker preferences for intervention in either direction $\left(\psi_{4} / \psi_{3}>>0\right.$ or $\left.\psi_{4} / \psi_{3}<<0\right)$ or strong risk aversion among informed traders $\left(\rho_{I}>>0\right)$ yield a unique equilibrium. Technically, these conditions guarantee that the right hand side of equilibrium condition (14) plotted in Figure 3 is very flat, so that it intersects the

\footnotetext{
${ }^{12}$ Section 2.3.4 on policy maker transparency below provides sufficient conditions on when the policy maker model exhibits a lower price informativeness than the standard model without, because making the policy maker information public (transparency) leads to the same price trading aggressiveness $a$ by traders as in the standard REE model without policy maker.
} 
$45^{\circ}$ line (left hand side of the equilibrium condition) only once and the equilibrium is unique. Intuitively, these conditions guarantee that a different policy maker reaction changes trader returns to information only slightly, thus the complementarity between trader aggressiveness $a$ and the policy maker reaction is weak. The proposition also proves uniqueness in the neighborhood of $\psi_{4} / \psi_{3}=0$, hence preferences for weak policy interventions do not induce equilibrium multiplicity.

The most important change compared to the previous section or models without policy maker is that the private information of the policy maker introduces additional risk for traders besides the usual risk about the asset fundamental $\theta$. Since this private information in part determines the policy maker's estimate of state $\theta$, and thus affects policy, there is additional policy risk in case of an informed policy maker.

Strong enough strategic complementarity can now induce multiple equilibria as follows. If informed traders increase $|a|$, i.e., trade more aggressively on information, then equilibrium prices become more informative. Consequently, the policy maker relies more on the price (rather than her private information) when inferring the state $\theta$ and making policy. This can be directly seen in the weight the policy maker places on her private information when making policy in (22) in the appendix. From the perspective of the traders, a smaller weight of private information on policy (and thus asset values) typically reduces the asset variance conditional on the price (see (17) in the appendix), because the risk introduced by the private information of the policy maker is reduced. Thus, larger $|a|$ induces a policy maker reaction that reduces the asset variance, which makes it more attractive to trade on information, i.e., increases $|a|$. Consequently, multiple equilibria with small or large $|a|$ may exist. This complementarity aspect does not exist in the standard CARA-normal models nor in the uninformed policy maker case, because there is no policy risk due to a privately informed policy maker. In Ozdenoren and Yuan (2008), feedback effects from prices to asset values also cause equilibrium multiplicity, but the mechanism is different, as multiplicity arises from a coordination motive between traders in their model.

\section{Proposition 5 (Equilibrium uniqueness).}

i. There exists $r^{*}>0$ such that, for all $\psi_{4} / \psi_{3}>r^{*}$, the linear equilibrium is unique with $a>0$.

ii. There exists $s^{*}<0$ such that, for all $\psi_{4} / \psi_{3}<s^{*}$, the linear equilibrium is unique with $a<0$.

iii. There exists $\rho^{*}>0$ such that, for all $\rho_{I}>\rho^{*}$, the linear equilibrium is unique.

iv. In the neighborhood of $\psi_{4} / \psi_{3}=0$, the linear equilibrium is unique.

Proof. See Appendix 3. 


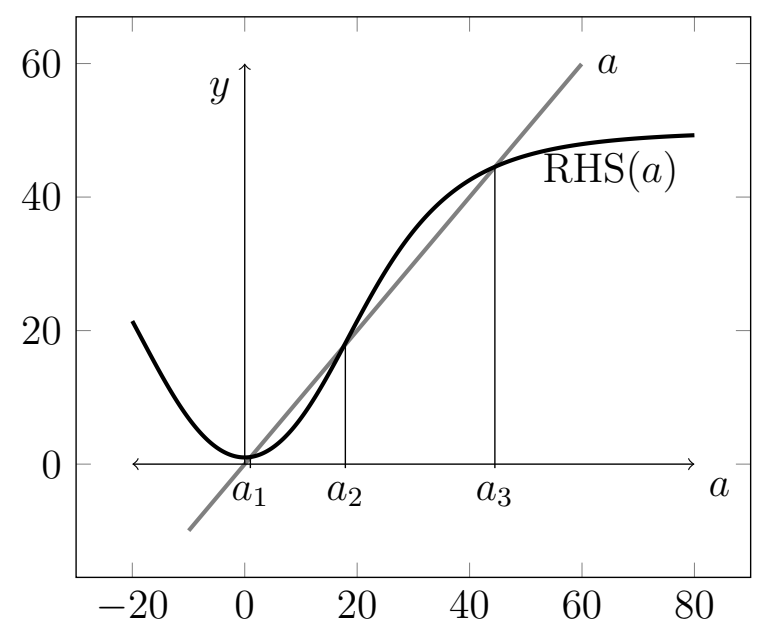

Figure 3: Equilibrium multiplicity. The graph plots the left hand side $(y=a)$ and right hand side $(y=\operatorname{RHS}(a))$ of equilibrium condition (14); the three intersections determine three different equilibrium values of $a$.

The proposition also tells us something about whether the equilibrium price function increases or decreases in the state. If the policy maker has preferences such that she increases asset values with larger fundamental $\theta\left(\psi_{4} / \psi_{3}>0\right)$, then informed traders will buy more (or sell less) assets with more positive information about the fundamental $(a>0)$. This also holds if $\psi_{4} / \psi_{3}<0$ is not too negative. Intuitively, $\psi_{4} / \psi_{3}<0$ close to zero means the policy maker reduces the asset value for a given $\theta$ compared to the standard model without policy maker, but the asset value is still increasing in $\theta$ after accounting for the policy component, since the policy maker does not intervene too strongly. For sufficiently negative $\psi_{4} / \psi_{3}$, however, the expected asset value is decreasing in $\theta$, since the policy component of the asset value decreases in $\theta$ and trumps the increasing fundamental component. Thus, the equilibrium trading aggressiveness $a$ in this case is negative, since a larger trader signal realization $s_{j}$ indicates a larger $\theta$-realization which indicates a lower asset value. The negative trading aggressiveness is in contrast to the case of an uninformed policy maker or the model without policy maker, where $a$ is always positive in equilibrium. This demonstrates how policy can affect trading strategies of traders, and is consistent with observations of the finance industry eagerly listening and reacting to announcements by central banks regarding policy changes, or with fast market reactions to ECB officials leaking information at private events the day before the announcement (e.g., Financial Times, 2015).

\subsubsection{Comparative statics}

Now we can turn to the question how the policy maker preferences affect price informativeness in equilibrium. The next proposition derives the comparative statics for the price informativeness for the cases where $\psi_{4} / \psi_{3}$ is either sufficiently positive or sufficiently negative, i.e., where the policy maker has strong preferences for intervention, which guarantees 
a unique equilibrium (Proposition 5). ${ }^{13}$ These are the most interesting cases, since $\psi_{4} / \psi_{3}$ close to zero implies that the optimal policy barely depends on state $\theta$. The proposition also derives the effect on price informativeness for positive and slightly negative values of $\psi_{4} / \psi_{3}$ if the equilibrium is unique.

For the purpose of comparative statics, I allow the quality of information for informed traders and policy maker to vary separately. $\tau_{\varepsilon}$ is the precision of the normally distributed trader signals as before, and $\tau_{\varepsilon}^{P}$ is the precision of the policy maker signal.

Similar to the case of an uninformed policy maker from above, define the price informativeness as difference of the mean squared errors of the policy maker estimates with and without the information from prices,

$$
\begin{aligned}
\mathrm{PI}_{\text {informed }}: & =\operatorname{MSE}\left(\mathbb{E}\left[\theta \mid s_{p}\right]\right)-\operatorname{MSE}\left(\mathbb{E}\left[\theta \mid p, s_{p}\right]\right)=\operatorname{Var}\left(\theta \mid s_{p}\right)-\operatorname{Var}\left(\theta \mid p, s_{p}\right) \\
& =\frac{1}{\left(\tau_{\theta}+\tau_{\varepsilon}^{P}\right)^{2} /\left((\mu a)^{2} \tau_{u}\right)+\tau_{\theta}+\tau_{\varepsilon}^{P}},
\end{aligned}
$$

which is increasing in trading aggressiveness $|a|$, share of informed traders $\mu$, noise precision $\tau_{u}$ and decreasing in prior precision $\tau_{\theta}$ and policy maker signal precision $\tau_{\varepsilon}^{P}$. Note that the price informativeness measure (15) refers to how informative prices are for the policy maker, not how informative prices are for uninformed outsiders, since the measure reflects the information gain from prices relative to the existing information $\left\{s_{p}\right\}$. This distinction allows me to analyze for example how a change in the policy maker signal precision affects the overall information that is available to the policy maker in equilibrium.

\section{Proposition 6 (Comparative statics).}

i. For policy preference parameter $\psi_{4} / \psi_{3}>0$ sufficiently large,

- decreasing $\psi_{4} / \psi_{3}$ increases price informativeness (15) for the policy maker in the unique linear equilibrium. Moreover,

- decreasing policy maker information precision $\tau_{\varepsilon}^{P}$, prior distribution precision $\tau_{\theta}$, or informed trader risk aversion $\rho_{I}$, and

- increasing the share of informed traders $\mu$, noise precision $\tau_{u}$, or trader signal precision $\tau_{\varepsilon}$

also increase price informativeness.

\footnotetext{
${ }^{13}$ It is possible to derive comparative statics under other conditions that guarantee uniqueness, but then the comparative statics may differ for $\psi_{4} / \psi_{3}<0$ close to zero and $\psi_{4} / \psi_{3}<<0$. It is also possible to derive comparative statics if there are multiple equilibria, but then comparative statics are equilibrium-specific and there typically exists at least one other equilibrium where the statics are reversed. If there are multiple equilibria as plotted in Figure 3 and if $\psi_{4} / \psi_{3}>0$, then the comparative statics results of $(i$.) carry over to all equilibria where the slope of the RHS is smaller than 1, i.e., the RHS crosses from above $\left(a_{1}\right.$ and $a_{3}$ in Figure 3), and are reversed for those where the RHS crosses from below $\left(a_{2}\right.$ in Figure 3 ). Due to this possible ambiguity of the comparative statics, the proposition focuses on cases where a unique equilibrium and unambiguous comparative statics are guaranteed.
} 
ii. For policy preference parameter $\psi_{4} / \psi_{3}<0$ sufficiently negative,

- increasing $\psi_{4} / \psi_{3}$ increases price informativeness in the unique linear equilibrium. Moreover,

- decreasing policy maker information precision $\tau_{\varepsilon}^{P}$, prior distribution precision $\tau_{\theta}$, or informed trader risk aversion $\rho_{I}$, and

- increasing the share of informed traders $\mu$, noise precision $\tau_{u}$, or trader signal precision $\tau_{\varepsilon}$

also increase price informativeness.

iii. In any unique equilibrium,

- increasing $\psi_{4} / \psi_{3}$ decreases price informativeness if $\psi_{4} / \psi_{3} \geq 0$ or if $\psi_{4} / \psi_{3}<0$ sufficiently close to zero, and

- increasing $\psi_{4} / \psi_{3}$ increases price informativeness if $\psi_{4} / \psi_{3}<0$ sufficiently negative.

Proof. See Appendix 3.

In short, more extreme policy maker preferences $\left(\psi_{4} / \psi_{3}\right)$ in either direction tend to decrease price informativeness, except in the mildly negative range of $\psi_{4} / \psi_{3}$, where preferences for slightly more negative intervention can increase price informativeness.

The intuition for these results is as follows. If $\psi_{4} / \psi_{3}>0$, then the policy maker has a preference for implementing policies that increase the asset value more for larger $\theta$, so that the asset value increases more steeply in $\theta$, since the asset value is $\theta+i\left(p, s_{p}\right)$. Thus, the policy maker intervention $i\left(p, s_{p}\right)$ amplifies the conditional asset value variance $\operatorname{Var}(\theta+$ $\left.i\left(p, s_{p}\right) \mid p, s_{j}\right)$ from the perspective of the traders. This variance increase has two components (see (17) in the proofs for the explicit expressions). First, a simple amplification as if the fundamental effect $\theta$ on the asset value is multiplied. Second, since the policy intervention is based in part on the private information of the policy maker, it adds additional risk from the perspective of the traders, since they do not observe the policy maker information. While both the trader signals as well as the policy maker signal are correlated with $\theta$, the noise term $\varepsilon_{p}$ in the policy maker signal $s_{p}=\theta+\varepsilon_{p}$ is unforecastable for traders which adds risk. Together, this additional "policy risk" leads the informed - who are risk averse - to trade less aggressively on their private information, i.e., $a>0$ decreases and price informativeness suffers. This is why more extreme policy maker preferences reduce price informativeness. ${ }^{14}$

Similarly, if $\psi_{4} / \psi_{3}<<0$, then the policy maker has a preference for implementing policies that decrease the asset value more for larger $\theta$, so reducing $\psi_{4} / \psi_{3}$ further amplifies

\footnotetext{
${ }^{14}$ There is a weaker, countervailing effect, because when the policy maker implements more extreme asset values, then the private information of traders is more valuable in forecasting the asset value, i.e., there is a larger linear return on information. Bond and Goldstein (2015) call this the "information importance effect", but it is outweighed by the quadratic "variance effect" for large $\left|\psi_{4} / \psi_{3}\right|$.
} 
the return variance and traders react by increasing $a<0$, i.e., also trade less aggressively and make prices less informative. In such an equilibrium, a lower price indicates a larger realization of $\theta$ due to the negative policy intervention, and traders use their information more to forecast the policy reaction than the fundamental part of the asset value, as the former dominates. Note that this $\psi_{4} / \psi_{3}<<0$ case amplifies the variance only for $\psi_{4} / \psi_{3}$ negative enough, as the policy reaction has to offset the fundamental effect of a larger $\theta$ on the asset value.

For mildly negative values of $\psi_{4} / \psi_{3}$, however, more extreme (i.e., more negative) values of $\psi_{4} / \psi_{3}$ can lower the return variance for traders, as the policy reaction acts like a random variable that is negatively correlated with the fundamental part $\theta$, which can offset some of the fundamental risk in the realization of $\theta$. Hence, in the negative range of $\psi_{4} / \psi_{3}$, slightly more extreme policy maker preferences can increase price informativeness. This case has interesting implications for policy maker transparency (see the next section).

While preferences of policy makers can usually not be chosen freely, it is important to recognize that policies with a large impact on asset values tend to make prices less informative. Hence, the comparative statics suggest that market information might be more useful for smaller policy interventions, i.e., actions that affect asset values not too strongly. In the context of banking regulation, for example, market information may help for minor policy purposes such as steering supervisors and monitoring activity in the right direction, ${ }^{15}$ but may not be as helpful for more extreme policy decisions such as bailouts. This may be a rather pessimistic result for proponents of market-based policy, as more information is typically most valuable in exactly these cases of extreme interventions where the market reveals less; this is certainly true in this model.

The comparative statics of the remaining exogenous parameters are unsurprising although the interpretation can be slightly different from the standard models due to equilibrium effects involving the policy maker. If the share of informed traders $\mu$ increases, then the informed trade more aggressively on information. This is because the price becomes more informative with more informed traders, and the policy maker reacts by placing a larger weight on the price rather than her private information when inferring the state $\theta$ and making her policy decision. This reduces the return variance from the perspective of the traders, because observing $p$ they can better infer the policy maker reaction. Similarly, less noise affecting the price (larger $\tau_{u}$ ) and better information for traders (larger $\tau_{\varepsilon}$ ) leads to the informed trading more aggressively, again because the policy maker uses her private information less in making policy, which reduces the variance from the perspective of the traders, i.e., reduces "policy risk." Finally, a smaller risk aversion $\rho_{I}$ leads to more aggressive trading and more informative prices, so the policy maker puts more weight on the

\footnotetext{
${ }^{15} \mathrm{As}$ is suggested often in the empirical banking literature, which finds that market prices of subordinated bank debt or bank equity prices can predict bank health or default, and may contain information that supervisors do not have (e.g., Berger et al., 2000; Gropp et al., 2006).
} 


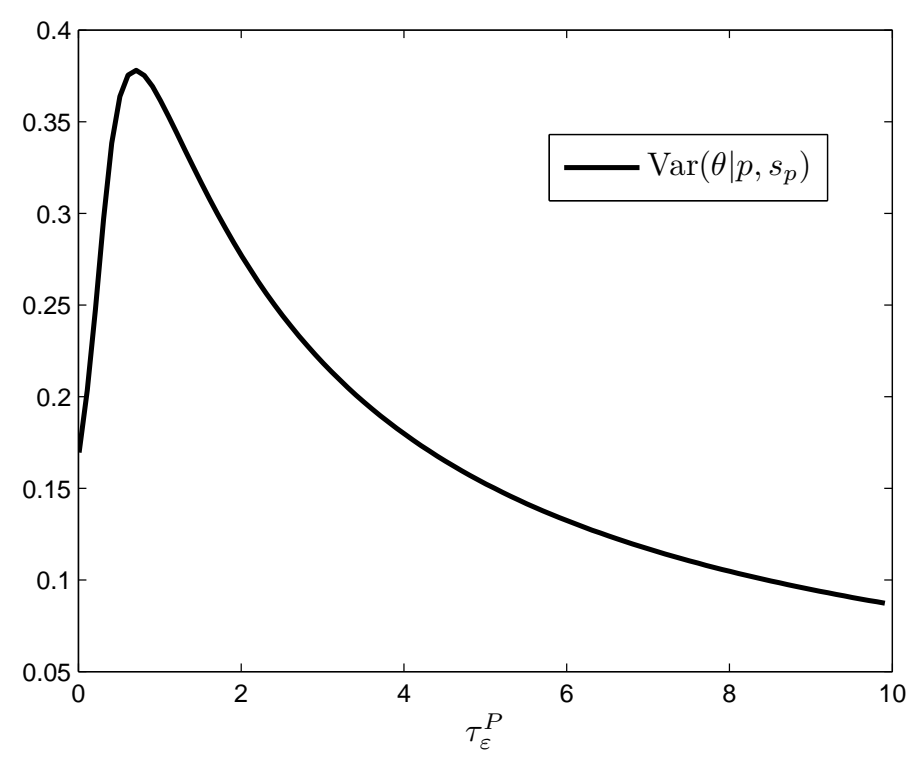

Figure 4: Plot of the variance of the policy maker estimate of the state $\theta$, depending on policy maker information precision $\tau_{\varepsilon}^{P}$ and taking into account equilibrium effects on price informativeness. The parameter values of the example are $\mu=1, \rho_{I}=1, \tau_{\theta}=1$, $\tau_{\varepsilon}=1, \tau_{u}=5, \psi_{4} / \psi_{3}=2$.

price when inferring the realization of $\theta$ (and less on her private information), which reduces policy risk for traders.

The effect of an increased precision of the policy maker information $\tau_{\varepsilon}^{P}$ on price informativeness is potentially ambiguous. On the one hand, more precise information means the policy maker signal $s_{p}=\theta+\varepsilon_{p}$ tends to be closer to the realization of $\theta$, which reduces risk from the perspective of the traders, as the error term $\varepsilon_{p}$ is unforecastable. On the other hand, more precise information means the policy maker relies more on her private information than the observable market prices, which increases risk for traders and could decrease $|a|$. The proof shows that a larger precision $\tau_{\varepsilon}^{P}$ increases $|a|$ for large enough $\left|\psi_{4} / \psi_{3}\right|$. However, the price informativeness measure (15) captures decreases in mean squared error due to market information relative to existing information $s_{p}$. And by increasing $\tau_{\varepsilon}^{P}$, the existing information becomes better, so there is a negative effect. The equilibrium effect of a larger $|a|$ does not offset this negative effect.

Interestingly, considering a different measure from price informativeness (15), the variance of the policy maker estimate $\operatorname{Var}\left(\theta \mid p, s_{p}\right)$, more policy maker information is not uniformly better. Taking equilibrium effects into account, i.e., how informed traders change $|a|$ and hence the informational content of market prices, better information for the policy maker (as measured by a lower policy maker signal variance $1 / \tau_{\varepsilon}^{P}$ ) can increase the variance of the policy maker estimate. Figure 4 plots one example, where the conditional variance is increasing in $\tau_{\varepsilon}^{P}$ for small values of $\tau_{\varepsilon}^{P}$, because the informed trade less aggressively on information $\left(|a|\right.$ decreases). However, the figure also shows that $\tau_{\varepsilon}^{P}$ decreases the conditional variance for large enough $\tau_{\varepsilon}^{P}$. Still, this has important implications for cases where the 
information quality of the policy maker $\tau_{\varepsilon}^{P}$ is costly and endogenous: Policy makers might choose not to acquire information, knowing that this would induce a larger information asymmetry between policy maker and traders, therefore increase policy risk and decrease information from the market.

\subsubsection{Policy maker transparency}

The comparative statics results showed that price informativeness can be strongly affected by the risk due to the policy maker action which affects asset values and variances. To reduce the policy risk - which exists solely because traders do not know the independent information of the policy maker $\left(s_{p}\right)$-and to make market prices more informative, the policy maker could make her information $s_{p}$ public ("transparency") prior to trading.

In this case, the variance of the asset value from the perspective of the traders changes from $\operatorname{Var}\left(\theta+i\left(p, s_{p}\right) \mid p, s_{j}\right)$ to $\operatorname{Var}\left(\theta+i\left(p, s_{p}\right) \mid p, s_{j}, s_{p}\right)=\operatorname{Var}\left(\theta \mid p, s_{j}, s_{p}\right)$. Thus, policy risk is completely removed, since traders know all the factors that determine policy, namely the prices $p$ and the policy maker's information $s_{p}$. This does not mean that the asset variance unambiguously decreases with transparency, but it does so for sufficiently extreme policy maker preferences $\left(\left|\psi_{4} / \psi_{3}\right|\right){ }^{16}$

If $s_{p}$ is publicly disclosed, then the equilibrium trading aggressiveness of the traders is $a=\tau_{\varepsilon} / \rho_{I}$, as in the standard model without policy maker or with an uninformed policy maker, since all policy risk is removed. This is why this section also serves as a comparison of price informativeness between the standard model and the policy maker model. If the policy maker does not disclose her information as assumed so far, then trading aggressiveness $a$ is implicitly defined by (14) as:

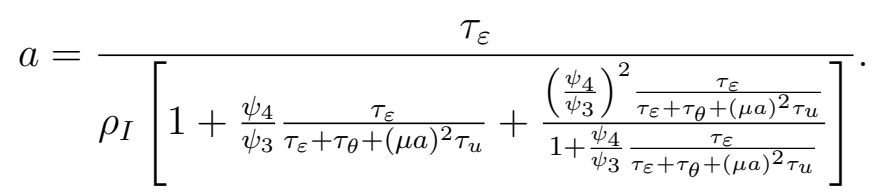

Clearly, for extreme policy maker preferences that increase or decrease the asset value a lot for any change in $\theta$ (i.e., large $\left|\psi_{4} / \psi_{3}\right|$ ), the equilibrium $|a|$ becomes very small (see also the proof of Proposition 5), because policy risk diminishes the incentives to trade on information for the risk averse traders. Indeed, $a \rightarrow 0$ as $\left|\psi_{4} / \psi_{3}\right| \rightarrow \infty$. Hence, a policy maker with preferences for strong interventions will be better off by disclosing her information, since it removes policy risk for traders and thereby makes prices more informative, as trading aggressiveness $a$ is the main determinant of price informativeness (15).

However, for policy makers with mild negative preferences $\left(\psi_{4} / \psi_{4}<0\right.$ close to zero), not revealing the information is better, as the policy action moves against the fundamental

\footnotetext{
${ }^{16}$ Too see this, substitute $\beta_{3}$ in $(22)$ into $\operatorname{Var}\left(\theta+i\left(p, s_{p}\right) \mid p, s_{j}\right)$ in (17). As $\left|\psi_{4} / \psi_{3}\right| \rightarrow \infty$, equilibrium effects cause $a \rightarrow 0$ (see (14)) and hence $\operatorname{Var}\left(\theta+i\left(p, s_{p}\right) \mid p, s_{j}\right) \rightarrow \infty$. The variance in case of transparency, on the other hand, is finite and given by $1 /\left(2 \tau_{\varepsilon}+\tau_{\theta}+\left(\mu \tau_{\varepsilon} / \rho_{I}\right)^{2} \tau_{u}\right)$.
} 
component $\theta$ of the asset value. Hence, a larger realization of $\theta$-which normally increases the asset value - tends to be offset to some degree by a counterveiling policy action reducing the asset value, thus reducing the asset value variance. In other words, policy "dampens" the fundamental $\theta$-shocks on the asset value, but this only works if the policy action (determined by $s_{p}$ ) is not already known at the time of trading. Because if $s_{p}$ is known, then traders already factor the policy reaction in at the time of trading and therefore policy cannot have have this dampening effect. Consequently, there are cases where transparency is detrimental to the policy maker interests (obtaining information from the financial market). The following proposition states and proves these results.

\section{Proposition 7 (Policy maker transparency).}

i. For $\left|\psi_{4} / \psi_{3}\right|$ sufficiently large, publishing the policy maker signal $s_{p}$ increases trading aggressiveness $|a|$ and price informativeness.

ii. For $\psi_{4} / \psi_{3}<0$ close to zero, there exists an equilibrium where publishing the policy maker signal decreases trading aggressiveness $|a|$ and price informativeness.

Proof. See Appendix 3.

Bond and Goldstein (2015) also discuss policy risk in their model (they call it "endogenous risk"), but find that the policy maker should never disclose her information $s_{p}$ about $\theta$, for then prices become completely uninformative. This is because the asset value in their model depends on $\theta$ only indirectly via policy, so once the policy is known, traders have no reason to use their information about $\theta$ to trade the asset. This is different here where the asset value both directly and indirectly (via policy) depends on the state $\theta$.

The question of transparency is probably most important in the context of central banking. Until the 1990s, intransparency and opacity was seen as a virtue by most central bankers (e.g., Blinder et al., 2008), at which point the question of transparency gained momentum both among policy makers and researchers as it might allow the central bank to affect market expectations and make policy more effective. Moreover, since the late 2000s, some central banks started using communications about future actions as policy instrument (forward guidance). This model only analyzes the policy maker and financial market interaction (and not the entire real economy) to understand whether the central bank can obtain information from the market. But even in this context, it has implications for central bank transparency.

First, if the central bank tries to obtain information from financial market prices, and if the central bank reaction to the state variable strongly affects asset values, then transparency is beneficial for the central bank (Proposition 7, i.). Geraats (2002) distinguishes several forms of central bank transparency such as political transparency (openness about policy objectives), economic transparency (openness about its information on economic conditions), or policy transparency (announcement of policy actions and indications of future 
policy). In the present model, transparency refers to making the policy maker signal about the state $\theta$ public, which in the most plausible interpretation is its information about the state of the economy. Yet the benefit of making this information public comes about not directly because it gives more information about the economy to traders. Instead, it is beneficial mainly because it removes policy risk, since this information determines policy. Thus, transparency in this model refers to both policy transparency and economic transparency, but with the former making the qualitative difference.

Second, given many good reasons for central bank transparency, one might ask "[I]s there any strong argument for the central bank not to reveal everything it knows?" (Reis, 2013). Proposition 7 (ii.) gives an argument. Since the policy can affect asset values, it could be used counter-cyclically to reduce the variance in asset returns in the model. But this only works if the central bank has information that traders do not have, so that policy can act as a random variable that is negatively correlated with the state realization, thus reducing asset value variance from the perspective of the traders. Interestingly, the mechanism at play here is often used as rationale in favor of transparency, because transparency reduces policy risk, but if policy is used counter-cyclically in the above sense, then intransparency may be more successful in reducing risk in some cases. And as a side effect, market prices are more informative for the policy maker.

\section{Concluding remarks}

This paper analyzed a setting where traders know something about a policy relevant variable $\theta$ that a policy maker does not know. Traders first trade an asset whose value depends on this variable $\theta$. Afterwards, the policy maker can observe the financial market prices and make inferences about $\theta$ depending on how informative prices are, and then determines her policy action which in turn affects asset values. This paper is the first to analyze under which conditions the financial market reveals policy-relevant information to the policy maker in a setting with noise and possible self-defeating prophecies.

In the case of an uninformed policy maker, who has no independent information to make policy except from what she infers from market prices, I derive a necessary and sufficient condition for the existence of a partially revealing equilibrium. Using the necessary and sufficient condition, one can easily determine for any policy maker utility function whether or not the policy maker can use market information in equilibrium. The intuition for why no revealing equilibrium may exist is the same as in the models without noise: The policy maker reaction to market prices changes asset values and thereby punishes traders for revealing their information, so that traders trying to predict the asset value face a self-defeating prophecy problem. While the uninformed policy maker objectives determine whether a revealing equilibrium exists, they do not affect how much information is revealed if an equilibrium exists. 
Interestingly, the Bernanke and Woodford (1997) non-revelation problem in their model without noise vanishes as soon as any amount of noise is introduced. Hence, paradoxically, noise can in some cases support information revelation by markets. This is because the noise prevents full revelation of all trader information, so that these retain incentives to trade on their information even if policy reacts to (some of) their information.

If the policy maker receives an imperfect signal about $\theta$ as independent information, then not only do the policy maker preferences affect whether a revealing equilibrium exists, but also how much information is revealed if an equilibrium exists. Policy maker objectives for strong interventions change asset values a lot and thus tend to decrease price informativeness, because the policy reaction amplifies the asset variance. Consequently, policy makers who prefer policies with large impact on asset values tend to get less information from the market for policy making.

The paper also addresses the question of policy maker transparency, i.e., whether a policy maker should reveal her information. Here the policy maker cares about information from the market so that she can make better policy decisions. Hence, transparency is optimal in case of extreme policy preferences, because transparency removes policy risk for risk averse traders and thereby boosts the incentives to trade on information. However, there are also cases where intransparency is optimal, for example if policy moves against fundamental shocks, so that policy dampens fundamental shocks from the perspective of the traders.

Several interesting questions follow from this analysis for future research. One question is how the results of this paper change if the policy maker not only sets policy but also trades in the financial market. Central banks conducting open market operations or governments owning public companies might be such policy makers, and the possibility to trade might add incentives to set policy in a way that increases trading profits. Since this might crowd out regular traders, this begs the question how prices are affected or to what degree policy makers change policy for profit in equilibrium. Moreover, the present setup did not allow the policy maker to have a preference over financial market prices, which might be interesting for policy advice on dealing with potential asset bubbles.

\section{Acknowledgements}

I am grateful to two anonymous referees, as well as Klaus Adam, Marco Bassetto, Johannes Bubeck, Antonio Cabrales, Jayant Ganguli, Hans Peter Grüner, Felix Jarman, Xavier Lambin, Stefan Niemann, Edward S. Prescott, Andreas Rapp, Xavier Vives, and seminar/conference participants at Mannheim, University College London, Tilburg, Nuffield, IESE, Rotterdam, Edinburgh, Essex, Surrey, Frankfurt School, Autonoma Barcelona, Carlos Madrid, BI Oslo, ENTER Jamboree 2015, VfS 2015 Münster, SAET 2015 Cambridge, EEA/ESEM 2016 Geneva, GEA 2016 Berlin for comments and discussion. This research was supported by the German Research Foundation (DFG) via SFB 884. 


\section{Appendix: Proofs}

Proof of Proposition 1. Conjecturing demand functions of the quasi-linear form in definition 2, the market clearing condition is (2). The market clearing condition has to hold for any realization of $(\theta, u)$, which appears directly only on the right hand side of $(2)$. Since $(\theta, u)$ appears on the left hand side only indirectly via the equilibrium price function $P(\theta, u)$, this price function must at least reveal the statistic $z=\theta+u /(\mu a)$, so that I now write $P(z)$. That is, all $(\theta, u)$ realizations with $p=P(\theta, u)$ must be on the line $z=\theta+u /(\mu a)$. Hence, any equilibrium price function reveals at least the realization of $z$ to the uninformed, which includes the policy maker. Below, I shall also confirm that the price function reveals at most $z$.

Using the information set for the uninformed $\{z\}$ and the information set for the informed $\left\{s_{j}, z\right\}$, both consisting exclusively of normally distributed signals, the asset values are normally distributed conditional on this information. Consequently, the optimal demand of the informed is uniquely determined and given by (3), which is of the conjectured quasilinear form. Matching coefficient $a, a=\tau_{\varepsilon} / \rho_{I}$. Matching $g_{I}$ yields (4). Similarly, the optimal demand for the uninformed is uniquely given by (5), and matching $g_{U}$ yields (6).

Substituting $g_{I}(p)$ and $g_{U}(p)$ into the market clearing condition (2) yields (7). Given that $P(z)$ is invertible, rewrite $P^{-1}(P(z))=z$ and, abusing notation, $i(P(z))=i(z)$, so that the market clearing condition can be rearranged for an explicit expression of $P(z)$ given in (8). Clearly, this price function is unique (given the assumption that $i(z)$ is unique), hence the equilibrium is unique in the class of quasi-linear equilibria if it exists.

Dropping constant factors and terms from the right hand side of (8), it is immediate that $P(z)$ is invertible as required if and only if (9) is invertible in $z$, so that an equilibrium exists. If (9) is non-invertible, it contradicts market clearing and no equilibrium exists.

It remains to be shown that the continuous equilibrium price function $P(\theta, u)$ does not reveal more than the realization of $z$, i.e., $P(\theta, u)$ depends on $(\theta, u)$ only via $\theta+u /(\mu a)$. Lemma 2 in Pálvölgyi and Venter (2015) shows this for the Grossman and Stiglitz (1980)model, and the proof can be applied directly to the problem here. For completeness, I will translate their proof into my notation.

I already established that all $(\theta, u)$ realizations for which $p=P(\theta, u)$ are on the line $\theta+u /(\mu a)$. Now, by contradiction, suppose $P(\theta, u)$ depends on $(\theta, u)$ not only via $z=$ $\theta+u /(\mu a)$. This implies there exist two pairs $\left(\theta_{1}, u_{1}\right) \neq\left(\theta_{2}, u_{2}\right)$ such that $z=\theta_{1}+u_{1} /(\mu a)=$ $\theta_{2}+u_{2} /(\mu a)$ with $P\left(\theta_{1}, u_{1}\right)=p_{1} \neq P\left(\theta_{2}, u_{2}\right)=p_{2}$. Given continuity of $P(\theta, u)$ (which is a defining property of the equilibrium class) and using the intermediate value theorem, we can find a pair $\left(\theta^{*}, u^{*}\right)$ with $\theta^{*}+u^{*} /(\mu a)=z$ and $P\left(\theta^{*}, u^{*}\right)=\left(p_{1}+p_{2}\right) / 2$ (e.g., increase $\theta$ and decrease $u)$. Similarly, we can find a pair $\left(\theta^{\prime}, u^{\prime}\right)$ with $\theta^{\prime}+u^{\prime} /(\mu a) \neq z$ and $P\left(\theta^{\prime}, u^{\prime}\right)=$ $\left(p_{1}+p_{2}\right) / 2$ (e.g., starting at $\min \left\{p_{1}, p_{2}\right\}$, by increasing $\theta$ and keeping $u$ constant). That is, we have two points with $P\left(\theta^{*}, u^{*}\right)=P\left(\theta^{\prime}, u^{\prime}\right)=\left(p_{1}+p_{2}\right) / 2$ and $\theta^{*}+u^{*} /(\mu a) \neq \theta^{\prime}+u^{\prime} /(\mu a)$. 
Yet this contradicts the previously established fact that all $(\theta, u)$ realizations for which $p=P(\theta, u)$ are on the same line $\theta+u /(\mu a)$. Consequently, a continuous price function $P(\theta, u)$ reveals exactly $z=\theta+u /(\mu a)$ in equilibrium.

Proof of Proposition 2. From (10), price informativeness does not depend on demand strategies except for coefficient $a$. From Proposition 1, the equilibrium strategy coefficient is $a=\tau_{\varepsilon} / \rho_{I}$. Thus, price informativeness is increasing in $|a|, \mu$ and $\tau_{u}$, and $|a|$ in turn is increasing in $\tau_{\varepsilon}$ and decreasing in $\rho_{I}$. Moreover, price informativeness (10) decreases in $\tau_{\theta}$.

Proof of Proposition 3. The solution approach for the noisy REE is the typical "guess and verify" approach: First, a conjecture about the shape of demand functions is made. In this case, we conjecture the demand functions to be linear in signal $s_{j}$ and price $p$, whichafter imposing market clearing - gives a linear price function $P(\theta, u)$ with undetermined coefficients. Second, according to this price conjecture, the price function $P(\theta, u)$ gives the relationship between state $\theta$ and price, which is used to update traders' beliefs about $\theta$ via Bayes' rule. Third, demand functions given the information sets are computed. Fourth, the undetermined coefficients are identified, which gives the actual relationship between $\theta$ and prices.

I am going to derive a symmetric linear noisy rational expectations equilibrium, where the conjecture is that traders use strategies

$$
X_{I}\left(p, s_{j}\right)=a s_{j}-c_{I} p+b_{I} \text { and } X_{U}(p)=-c_{U} p+b_{U},
$$

which yields the market clearing condition

$$
\begin{array}{r}
\int_{0}^{\mu}\left(a s_{j}-c_{I} p+b_{I}\right) d j+\int_{\mu}^{1}\left(-c_{U} p+b_{U}\right) d j+u=0 \\
\Longleftrightarrow p=\frac{\mu a \theta+\mu b_{I}+(1-\mu) b_{U}+u}{\mu c_{I}+(1-\mu) c_{U}}
\end{array}
$$

because an appropriate law of large numbers for i.i.d. random variables (Sun, 2006) yields $\int_{0}^{\mu} s_{j} d j=\mu \theta$. Define $\lambda:=\left(\mu c_{I}+(1-\mu) c_{U}\right)^{-1}$ and $\tilde{b}:=\mu b_{I}+(1-\mu) b_{U}$ to simplify notation, and rearrange to obtain

$$
\frac{p-\lambda \tilde{b}}{\lambda \mu a}=\theta+u /(\mu a) .
$$

The left hand side is informationally equivalent to the market price $p$, since it just subtracts a constant and divides by another constant, and the equality implies that the equilibrium price function reveals the linear statistic "state $\theta$ plus a normally distributed noise term $u /(\mu a)^{\prime}$.

The variance of the net asset value for informed traders conditional on their information 


$$
\begin{aligned}
\operatorname{Var}\left(\theta+i\left(p, s_{p}\right)-p \mid p, s_{j}\right)= & \operatorname{Var}\left(\theta+i\left(p, s_{p}\right) \mid p, s_{j}\right)=\operatorname{Var}\left(\theta+\beta_{1}+\beta_{2} p+\beta_{3} s_{p} \mid p, s_{j}\right) \\
& =\operatorname{Var}\left(\theta\left(1+\beta_{3}\right)+\beta_{3} \varepsilon \mid p, s_{j}\right)=\frac{\left(1+\beta_{3}\right)^{2}}{\tau_{\varepsilon}+\tau_{\theta}+(\mu a)^{2} \tau_{u}}+\frac{\beta_{3}^{2}}{\tau_{\varepsilon}},
\end{aligned}
$$

since $\operatorname{Var}\left(\theta \mid p, s_{j}\right)=1 /\left(\tau_{\varepsilon}+\tau_{\theta}+(\mu a)^{2} \tau_{u}\right)$, as in the standard model without policy maker, and $\theta$ and $\varepsilon$ are independent. Similarly, for the uninformed, the variance of the asset value given the price is

$$
\operatorname{Var}\left(\theta+i\left(p, s_{p}\right)-p \mid p\right)=\frac{\left(1+\beta_{3}\right)^{2}}{\tau_{\theta}+(\mu a)^{2} \tau_{u}}+\frac{\beta_{3}^{2}}{\tau_{\varepsilon}}
$$

The Bayesian updating rule for the mean of normal distributions is a precision weighted sum of prior mean and signals, where the precision of the price signal is the inverse of its conditional variance, hence the conditional expectation of the asset value is

$$
\begin{array}{r}
\left.\left.\mathbb{E}\left[\theta+i\left(p, s_{p}\right)-p\right) \mid p, s_{j}\right]=\mathbb{E}\left[\theta\left(1+\beta_{3}\right)+\beta_{3} \varepsilon\right) \mid p, s_{j}\right]+\beta_{1}+\beta_{2} p-p \\
=\left(1+\beta_{3}\right) \frac{\tau_{\theta} \bar{\theta}+\tau_{\varepsilon} s_{j}+(\mu a)^{2} \tau_{u}\left(\frac{p-\lambda \tilde{b}}{\lambda \mu a}\right)}{\tau_{\theta}+\tau_{\varepsilon}+(\mu a)^{2} \tau_{u}}+\beta_{1}+\beta_{2} p-p
\end{array}
$$

Similarly, the expectation of the asset value for uninformed traders is

$$
\left.\mathbb{E}\left[\theta+i\left(p, s_{p}\right)-p\right) \mid p\right]=\left(1+\beta_{3}\right) \frac{\tau_{\theta} \bar{\theta}+(\mu a)^{2} \tau_{u}\left(\frac{p-\lambda \tilde{b}}{\lambda \mu a}\right)}{\tau_{\theta}+(\mu a)^{2} \tau_{u}}+\beta_{1}+\beta_{2} p-p .
$$

The well-known CARA demand functions derived from the first order conditions are given by

$$
\begin{aligned}
X_{I}\left(p, s_{j}\right) & =\frac{\left.\mathbb{E}\left[\theta+i\left(p, s_{p}\right)-p\right) \mid p, s_{j}\right]}{\rho_{I} \operatorname{Var}\left(\theta+i\left(p, s_{p}\right)-p \mid p, s_{j}\right)}, \\
X_{U}(p) & =\frac{\left.\mathbb{E}\left[\theta+i\left(p, s_{p}\right)-p\right) \mid p\right]}{\rho_{U} \operatorname{Var}\left(\theta+i\left(p, s_{p}\right)-p \mid p\right)} .
\end{aligned}
$$

Note that the trader objective is concave even if $i(p)$ is highly convex, because a single trader does not affect $p$, hence $i(p)$ is a constant in the trader maximization problem. Plugging in for conditional expectations and variances,

$$
X_{U}(p)=\frac{\left(1+\beta_{3}\right) \frac{\tau_{\theta} \bar{\theta}+(\mu a)^{2} \tau_{u}\left(\frac{p-\lambda \tilde{b}}{\lambda \mu a}\right)}{\tau_{\theta}+(\mu a)^{2} \tau_{u}}+\beta_{1}+\beta_{2} p-p}{\rho_{U}\left[\frac{\left(1+\beta_{3}\right)^{2}}{\tau_{\theta}+(\mu a)^{2} \tau_{u}}+\frac{\beta_{3}^{2}}{\tau_{\varepsilon}}\right]}
$$

which is linear in $p$ as conjectured, hence identifying coefficients of the linear demand func- 
tion $X_{U}(p)=-c_{U} p+b_{U}$, we obtain

$$
c_{U}=\frac{1-\beta_{2}-\frac{\left(1+\beta_{3}\right) \mu a \tau_{u} / \lambda}{\tau_{\theta}+(\mu a)^{2} \tau_{u}}}{\rho_{U}\left[\frac{\left(1+\beta_{3}\right)^{2}}{\tau_{\theta}+(\mu a)^{2} \tau_{u}}+\frac{\beta_{3}^{2}}{\tau_{\varepsilon}}\right]}, b_{U}=\frac{\beta_{1}+\frac{\left(1+\beta_{3}\right)\left(\tau_{\theta} \bar{\theta}-\mu a \tau_{u} \tilde{b}\right)}{\tau_{\theta}+(\mu a)^{2} \tau_{u}}}{\rho_{U}\left[\frac{\left(1+\beta_{3}\right)^{2}}{\tau_{\theta}+(\mu a)^{2} \tau_{u}}+\frac{\beta_{3}^{2}}{\tau_{\varepsilon}}\right]} .
$$

For informed traders, plugging in for conditional expectations and variances,

$$
X_{I}\left(p, s_{j}\right)=\frac{\left(1+\beta_{3}\right) \frac{\tau_{\theta} \bar{\theta}+\tau_{\varepsilon} s_{j}+(\mu a)^{2} \tau_{u}\left(\frac{p-\lambda \tilde{b}}{\lambda \mu a}\right)}{\tau_{\theta}+\tau_{\varepsilon}+(\mu a)^{2} \tau_{u}}+\beta_{1}+\beta_{2} p-p}{\rho_{I}\left[\frac{\left(1+\beta_{3}\right)^{2}}{\tau_{\varepsilon}+\tau_{\theta}+(\mu a)^{2} \tau_{u}}+\frac{\beta_{3}^{2}}{\tau_{\varepsilon}}\right]},
$$

which is linear in signal $s_{j}$ and price $p$ as conjectured. Matching coefficients of $X_{I}\left(p, s_{j}\right)=$ $a s_{j}-c_{I} p+b_{I}$,

$$
\begin{gathered}
a=\frac{\left(1+\beta_{3}\right) \tau_{\varepsilon}}{\rho_{I}\left(\tau_{\theta}+\tau_{\varepsilon}+(\mu a)^{2} \tau_{u}\right)\left[\frac{\left(1+\beta_{3}\right)^{2}}{\tau_{\varepsilon}+\tau_{\theta}+(\mu a)^{2} \tau_{u}}+\frac{\beta_{3}^{2}}{\tau_{\varepsilon}}\right]}, \\
c_{I}=\frac{1-\beta_{2}-\frac{\left(1+\beta_{3}\right) \mu a \tau_{u} / \lambda}{\tau_{\theta}+\tau_{\varepsilon}+(\mu a)^{2} \tau_{u}}}{\rho_{I}\left[\frac{\left(1+\beta_{3}\right)^{2}}{\tau_{\varepsilon}+\tau_{\theta}+(\mu a)^{2} \tau_{u}}+\frac{\beta_{3}^{2}}{\tau_{\varepsilon}}\right]}, \quad b_{U}=\frac{\beta_{1}+\frac{\left(1+\beta_{3}\right)\left(\tau_{\theta} \bar{\theta}-\mu a \tau_{u} \tilde{b}\right)}{\tau_{\theta}+\tau_{\varepsilon}+(\mu a)^{2} \tau_{u}}}{\rho_{I}\left[\frac{\left(1+\beta_{3}\right)^{2}}{\tau_{\varepsilon}+\tau_{\theta}+(\mu a)^{2} \tau_{u}}+\frac{\beta_{3}^{2}}{\tau_{\varepsilon}}\right]} .
\end{gathered}
$$

Now both $c_{I}$ and $c_{U}$ depend on $\lambda$ and vice versa. Substitute $c_{I}$ and $c_{U}$ into $\lambda=1 /\left(\mu c_{I}+\right.$ $\left.(1-\mu) c_{U}\right)$ and solve for $\lambda$ to get

$$
\lambda=\frac{1+\frac{\left(1+\beta_{3}\right) \mu^{2} a \tau_{u}}{\rho_{I}\left(\tau_{\theta}+\tau_{\varepsilon}+(\mu a)^{2} \tau_{u}\right)\left[\frac{\left(1+\beta_{3}\right)^{2}}{\tau_{\varepsilon}+\tau_{\theta}+(\mu a)^{2} \tau_{u}}+\frac{\beta_{3}^{2}}{\tau_{\varepsilon}}\right]}+\frac{\left(1+\beta_{3}\right) \mu(1-\mu) \tau_{u}}{\rho_{U}\left(\tau_{\theta}+(\mu a)^{2} \tau_{u}\right)\left[\frac{\left(1+\beta_{3}\right)^{2}}{\tau_{\theta}+(\mu a)^{2} \tau_{u}}+\frac{\beta_{3}^{2}}{\tau_{\varepsilon}}\right]}}{\frac{\mu\left(1-\beta_{2}\right)}{\rho_{I}\left[\frac{\left(1+\beta_{3}\right)^{2}}{\tau_{\varepsilon}+\tau_{\theta}+(\mu a)^{2} \tau_{u}}+\frac{\beta_{3}^{2}}{\tau_{\varepsilon}}\right]}+\frac{(1-\mu)\left(1-\beta_{2}\right)}{\rho_{U}\left[\frac{\left(1+\beta_{3}\right)^{2}}{\tau_{\theta}+(\mu a)^{2} \tau_{u}}+\frac{\beta_{3}^{2}}{\tau_{\varepsilon}}\right]}} .
$$

Furthermore, both $b_{I}$ and $b_{U}$ depend on $\tilde{b}$ and vice versa. Substitute both into $\tilde{b}=\mu b_{I}+$ $(1-\mu) b_{U}$ and solve for $\tilde{b}$ to get

$$
\tilde{b}=\frac{\mu\left[\frac{\frac{\left(1+\beta_{3}\right) \tau_{\theta} \bar{\theta}}{\tau_{\theta}+\tau_{\varepsilon}+(\mu a)^{2} \tau_{u}}+\beta_{1}}{\rho_{I}\left[\frac{\left(1+\beta_{3}\right)^{2}}{\tau_{\varepsilon}+\tau_{\theta}+(\mu a)^{2} \tau_{u}}+\frac{\beta_{3}^{2}}{\tau_{\varepsilon}}\right]}\right]+(1-\mu)\left[\frac{\frac{\left(1+\beta_{3}\right) \tau_{\theta} \bar{\theta}}{\tau_{\theta}+(\mu a)^{2} \tau_{u}}+\beta_{1}}{\rho_{U}\left[\frac{\left(1+\beta_{3}\right)^{2}}{\tau_{\theta}+(\mu a)^{2} \tau_{u}}+\frac{\beta_{3}^{2}}{\tau_{\varepsilon}}\right]}\right]}{1+\frac{(1-\mu) \mu a \tau_{u}\left(1+\beta_{3}\right)}{\rho_{I}\left(\tau_{\theta}+\tau_{\varepsilon}+(\mu a)^{2} \tau_{u}\right)\left[\frac{\left(1+\beta_{3}\right)^{2}}{\tau_{\varepsilon}+\tau_{\theta}+(\mu a)^{2} \tau_{u}}+\frac{\beta_{3}^{2}}{\tau_{\varepsilon}}\right]}+\frac{\rho_{U}\left(\tau_{\theta}+(\mu a)^{2} \tau_{u}\right)\left[\frac{\left(1+\beta_{3}\right)^{2}}{\tau_{\theta}+(\mu a)^{2} \tau_{u}}+\frac{\beta_{3}^{2}}{\tau_{\varepsilon}}\right]}{\rho_{\varepsilon}}} .
$$

These terms still depend on the endogenous objects $a, \beta_{1}, \beta_{2}, \beta_{3}$. Turning to the policy maker, her utility function (11) is strictly concave by construction, so the first order condition determines the unique reaction function

$$
i\left(p, s_{p}\right)=\frac{\psi_{2}+\psi_{4} \mathbb{E}\left[\theta \mid p, s_{p}\right]}{\psi_{3}}=\frac{\psi_{2}+\psi_{4} \frac{\tau_{\theta} \bar{\theta}+\tau_{\varepsilon} s_{p}+(\mu a)^{2} \tau_{u}\left(\frac{p-\lambda \tilde{b}}{\mu a \lambda}\right)}{\tau_{\theta}+\tau_{\varepsilon}+(\mu a)^{2} \tau_{u}}}{\psi_{3}} .
$$


Matching coefficients for $i\left(p, s_{p}\right)=\beta_{1}+\beta_{2} p+\beta_{3} s_{p}$, we obtain

$$
\beta_{1}=\frac{\psi_{2}+\psi_{4} \frac{\tau_{\theta} \bar{\theta}-\mu a \tau_{u} \tilde{b}}{\tau_{\theta}+\tau_{\varepsilon}+(\mu a)^{2} \tau_{u}}}{\psi_{3}}, \beta_{2}=\frac{\psi_{4} \frac{\mu a \tau_{u} / \lambda}{\tau_{\theta}+\tau_{\varepsilon}+(\mu a)^{2} \tau_{u}}}{\psi_{3}}, \beta_{3}=\frac{\psi_{4} \frac{\tau_{\varepsilon}}{\tau_{\theta}+\tau_{\varepsilon}+(\mu a)^{2} \tau_{u}}}{\psi_{3}} .
$$

Substitute the term for $\beta_{3}$ in (22) into coefficient $a(19)$, so that the equilibrium condition for $a$ is (14), which depends only on one endogenous strategy variable $(a)$. The condition can be rearranged as a fifth degree polynomial in $a$. Fifth degree polynomials are guaranteed to have at least one solution and at most five. Given a solution for $a, \beta_{3}$ in (22) is uniquely determined. Given $\beta_{3}$ and $a$, substituting $\beta_{2}$ into $\lambda$ in (20) yields a linear condition with a unique solution for $\lambda$, which in turn determines $\beta_{2}$ in (22) and $c_{U}, c_{I}$ in (18), (19) uniquely. Substituting $\beta_{1}$ into $\tilde{b}$ in (21) yields a linear condition with a unique solution for $\tilde{b}$, which in turn determines $\beta_{1}$ uniquely. Finally, for equilibrium, market clearing requires $\mu c_{I}+(1-$ $\mu) c_{U} \neq 0$ with the coefficients $c_{I}, c_{U}$ just obtained. Thus, an equilibrium exists for almost all parameter profiles except where $\mu c_{I}+(1-\mu) c_{U}=0$, and $a$ is the only source of equilibrium multiplicity (i.e., all other endogenous variables are uniquely determined given $a$ ).

\section{Proof of Corollary 4.}

$i$. Setting $a=0$ in equilibrium condition (14) yields a contradiction for any $\tau_{\varepsilon}>0$.

ii. The right hand side of (14) is positive for any $a \in \mathbb{R}$ if $\frac{\psi_{4}}{\psi_{3}}>-\frac{\tau_{\varepsilon}+\tau_{\theta}}{\tau_{\varepsilon}}$, hence any solution to (14) must be $a>0$.

\section{Proof of Proposition 5.}

$i$. Using the shorthand $r:=\psi_{4} / \psi_{3}$, recall equilibrium condition (14):

$$
a=\frac{\tau_{\varepsilon}}{\rho_{I}\left[1+r \frac{\tau_{\varepsilon}}{\tau_{\varepsilon}+\tau_{\theta}+(\mu a)^{2} \tau_{u}}+\frac{r^{2} \frac{\tau_{\varepsilon}}{\tau_{\varepsilon}+\tau_{\theta}+(\mu a)^{2} \tau_{u}}}{1+r \frac{\tau_{\varepsilon}}{\tau_{\varepsilon}+\tau_{\theta}+(\mu a)^{2} \tau_{u}}}\right]}
$$

Every $a$ that fulfills condition (14) forms an equilibrium; uniqueness requires a single intersection of $a$ and the right hand side (RHS) of the condition. Note that the RHS of (14) is symmetric about $a=0$, since $a$ enters the condition only quadratically. Note also that the RHS of (14) is positive for all $a \geq 0$ at all $r>0$. Using the shorthand $\tau:=\tau_{\varepsilon}+\tau_{\theta}+(\mu a)^{2} \tau_{u}$, the slope of the RHS of (14) in $a$ is

$$
\frac{\partial \operatorname{RHS}(a)}{\partial a}=\tau_{\varepsilon} \rho_{I}^{-1}\left[2 r \tau_{\varepsilon} \tau_{u} \mu^{2} a \tau^{-2}+2 r^{2} \tau_{\varepsilon} \tau_{u} \mu^{2} a\left(\tau+r \tau_{\varepsilon}\right)^{-2}\right] /\left[1+r \frac{\tau_{\varepsilon}}{\tau}+\frac{r^{2} \tau_{\varepsilon}}{\tau+r \tau_{\varepsilon}}\right]^{2}
$$

This slope is positive for $r>0$ and approaches zero as $r \rightarrow \infty$ at all $a>0$, since the denominator grows at a quadratic rate in $r$, while the numerator only grows at a linear rate. Now, at $a=0, \operatorname{RHS}(a)>a=0$. Since a large $r$ makes the slope of $\operatorname{RHS}(a)$ arbitrarily small, there exists some $r^{*}>0$ such that the slope of $\operatorname{RHS}(a)$ is less than 1 
for all $r \geq r^{*}$, which guarantees that $\operatorname{RHS}(a)$ crosses $a$ exactly once in $a \geq 0$, i.e., (14) has only one non-negative solution. It remains to be shown that there is no further solution $a=\operatorname{RHS}(a)$ in $a<0$. This immediately follows from the fact that $\operatorname{RHS}(a)>0$ for all $a \in \mathbb{R}$ if $r>0$.

ii. We want to show that there exists $s^{*}<0$ such that $r<s^{*}$ guarantees a unique solution to (14), and that this solution is $a<0$. The slope of the RHS (23) approaches zero as $r \rightarrow-\infty$ at all $a \in \mathbb{R}$, since the denominator grows at a quadratic rate whereas the numerator changes only at a linear rate in $r$. Thus, there exists some $s^{*}<0$ such that the slope of $\operatorname{RHS}(a)$ is smaller than 1 for all $r \leq s^{*}$, which guarantees that the RHS intersects $a$ exactly once.

The RHS of the equilibrium condition (14) is negative at $a=0$ if and only if $r<-\frac{\tau_{\varepsilon}+\tau_{\theta}}{\tau_{\varepsilon}}$. Moreover, $\operatorname{RHS}(a)$ converges to a positive constant as $a \rightarrow-\infty$. Hence, the unique intersection fulfilling (14) must be at $a<0$.

iii. The slope of the RHS in a given in (23) approaches zero as $\rho_{I} \rightarrow \infty$. The same arguments as in (i.) and (ii.) imply that we can find a threshold $\rho^{*}>0$ such that, for all $\rho_{I}>\rho^{*}$, the slope of the RHS is less than 1 everywhere, which guarantees a unique equilibrium.

$i v$. Clearly, for $\psi_{4} / \psi_{3}=0,(14)$ simplifies to $a=\tau_{\varepsilon} / \rho_{I}$ and the slope of the RHS in $a$ is zero. Since the RHS of (14) is continuously differentiable in $\psi_{4} / \psi_{3}$ and $a$, the slope of the RHS (23) is close to zero for any $\psi_{4} / \psi_{3}$ close to zero. Hence, in that neighborhood there can be only one intersection of the RHS with $a$, i.e., only one solution to (14).

Proof of Proposition 6. We can rewrite the equilibrium condition (14), which implicitly defines $a$, as $\operatorname{RHS}(a, t)-a=0$, where $\operatorname{RHS}(a, t)$ is the right hand side of (14) as a function of $a$ and parameter $t$. By the implicit function theorem, we can determine how $a$ changes in equilibrium in response to a small change in parameter $t$ :

$$
\frac{\partial a}{\partial t}=-\frac{\mathrm{RHS}_{t}}{\mathrm{RHS}_{a}-1}
$$

where $\mathrm{RHS}_{t}$ is the partial derivative with respect to $t$. The uniqueness proofs of Proposition 5 show that $\mathrm{RHS}_{a}$ is less than 1 for $\left|\psi_{4} / \psi_{3}\right|$ large enough, hence it follows that $a$ increases if and only if $\mathrm{RHS}_{t}>0$ and decreases if and only if $\mathrm{RHS}_{t}<0$.

$i$. Using the shorthands $r:=\psi_{4} / \psi_{3}$ and $\tau:=\tau_{\varepsilon}+\tau_{\theta}+(\mu a)^{2} \tau_{u}$, and applying the quotient rule twice to the right hand side of (14),

$$
\operatorname{RHS}_{r}=\underbrace{-\tau_{\varepsilon} \rho_{I}^{-1}}_{<0} \underbrace{\left[1+r \tau_{\varepsilon} / \tau+\frac{r^{2} \tau_{\varepsilon} / \tau}{1+r \tau_{\varepsilon} / \tau}\right]^{-2}}_{>0}\left[\tau_{\varepsilon} / \tau+\frac{2 r \tau_{\varepsilon} / \tau+r^{2}\left(\tau_{\varepsilon} / \tau\right)^{2}}{\left(1+r \tau_{\varepsilon} / \tau\right)^{2}}\right]
$$


which is negative if and only if the term in the last bracket is positive. That last term is positive since $r>0$ by assumption and all other parameters are positive. Thus, $\mathrm{RHS}_{r}<0$ and $a$ decreases with larger $r$.

Next, $\mathrm{RHS}_{\mu}>0$ for $r>0$ :

$$
\operatorname{RHS}_{\mu}=\underbrace{-\tau_{\varepsilon} \rho_{I}^{-1}}_{<0} \underbrace{\left[1+r \tau_{\varepsilon} / \tau+\frac{r^{2} \tau_{\varepsilon} / \tau}{1+r \tau_{\varepsilon} / \tau}\right]^{-2}}_{>0} \underbrace{\left[-2 r \tau_{\varepsilon} a^{2} \mu \tau_{u} / \tau^{2}-2 r^{2} \tau_{\varepsilon} a^{2} \mu \tau_{u} /\left(\tau+r \tau_{\varepsilon}\right)^{2}\right]}_{<0 \text { if } r>0} .
$$

Moreover, $\mathrm{RHS}_{\tau_{\theta}}>0$ :

$$
\operatorname{RHS}_{\tau_{\theta}}=\underbrace{-\tau_{\varepsilon} \rho_{I}^{-1}}_{<0} \underbrace{\left[1+r \tau_{\varepsilon} / \tau+\frac{r^{2} \tau_{\varepsilon} / \tau}{1+r \tau_{\varepsilon} / \tau}\right]^{-2}}_{>0} \underbrace{\left[-r \tau_{\varepsilon} / \tau^{2}-r^{2} \tau_{\varepsilon} /\left(\tau+r \tau_{\varepsilon}\right)^{2}\right]}_{<0 \text { if } r>0} .
$$

It can similarly be verified that $\mathrm{RHS}_{\rho_{I}}<0$ and $\mathrm{RHS}_{\tau_{u}}>0$.

To investigate the effects of $\tau_{\varepsilon}$ and $\tau_{\varepsilon}^{P}$ separately, we have to derive the equilibrium condition for $a$ again, which is more complicated in the more general case:

$$
a=\frac{\left(1+r \frac{\tau_{\varepsilon}^{P}}{\tau_{\theta}+\tau_{\varepsilon}^{P}+(\mu a)^{2} \tau_{u}}\right) \tau_{\varepsilon}}{\rho_{I}\left[\left(1+r \frac{\tau_{\varepsilon}^{P}}{\tau_{\theta}+\tau_{\varepsilon}^{P}+(\mu a)^{2} \tau_{u}}\right)^{2}+\left(\tau_{\theta}+\tau_{\varepsilon}+(\mu a)^{2} \tau_{u}\right)\left(r \frac{\tau_{\varepsilon}^{P}}{\tau_{\theta}+\tau_{\varepsilon}^{P}+(\mu a)^{2} \tau_{u}}\right)^{2} / \tau_{\varepsilon}^{P}\right]} .
$$

This is also a fifth degree polynomial in $a$, guaranteeing a solution. A tedious but straightforward computation using the quotient rule shows that the slope of the RHS in $a$ gets arbitrarily close to zero for $|r| \rightarrow \infty$, since the denominator of $\mathrm{RHS}_{a}$ grows at a quartic rate while numerator grows only quadratically, thus we also have uniqueness for $|r|$ large enough in this more general case. It remains to determine the sign of the slope of the RHS in $\tau_{\varepsilon}$ and $\tau_{\varepsilon}^{P}$ for the comparative statics. Abbreviating $\beta_{3}=r \frac{\tau_{\varepsilon}^{P}}{\tau_{\theta}+\tau_{\varepsilon}^{P}+(\mu a)^{2} \tau_{u}}$,

$$
\begin{aligned}
\frac{\partial \operatorname{RHS}(28)}{\partial \tau_{\varepsilon}} & =\frac{\left.\left(1+\beta_{3}\right) \rho_{I}\left[\left(1+\beta_{3}\right)^{2}+\tau \beta_{3}^{2} / \tau_{\varepsilon}^{P}\right)\right]-\left(1+\beta_{3}\right) \tau_{\varepsilon} \rho_{I} \beta_{3}^{2} / \tau_{\varepsilon}^{P}}{\left[\rho_{I}\left(\left(1+\beta_{3}\right)^{2}+\tau \beta_{3}^{2} / \tau_{\varepsilon}^{P}\right)\right]^{2}} \\
& =\frac{\left.\left(1+\beta_{3}\right) \rho_{I}\left[\left(1+\beta_{3}\right)^{2}+\left(\tau_{\theta}+(\mu a)^{2} \tau_{u}\right) \beta_{3}^{2} / \tau_{\varepsilon}^{P}\right)\right]}{\left[\rho_{I}\left(\left(1+\beta_{3}\right)^{2}+\tau \beta_{3}^{2} / \tau_{\varepsilon}^{P}\right)\right]^{2}}>0,
\end{aligned}
$$

since $r>0$ implies $\beta_{3}>0$, thus $a>0$ in equilibrium increases with an increase of $\tau_{\varepsilon}$. 
Finally, denoting $\beta_{3}^{\prime}=\partial \beta_{3} / \partial \tau_{\varepsilon}^{P}=r \frac{\tau_{\theta}+(\mu a)^{2} \tau_{u}}{\left(\tau_{\varepsilon}^{P}+\tau_{\theta}+(\mu a)^{2} \tau_{u}\right)^{2}}>0$,

$$
\begin{array}{r}
\frac{\partial \mathrm{RHS}(28)}{\partial \tau_{\varepsilon}^{P}}>0 \Longleftrightarrow \beta_{3}^{\prime} \tau_{\varepsilon} \rho_{I}\left[\left(1+\beta_{3}\right)^{2}+\tau \beta_{3}^{2} / \tau_{\varepsilon}^{P}\right] \\
-\left(1+\beta_{3}\right) \tau_{\varepsilon} \rho_{I}\left[2\left(1+\beta_{3}\right) \beta_{3}^{\prime}+\tau\left(2 \beta_{3} \beta_{3}^{\prime} \tau_{\varepsilon}^{P}-\beta_{3}^{2}\right) / \tau_{\varepsilon}^{P^{2}}\right] \\
=-\left(\tau_{\varepsilon} \rho_{I}\left(1+\beta_{3}\right)^{2} \beta_{3}^{\prime}+\tau_{\varepsilon} \rho_{I} \tau 2 \beta_{3} \beta_{3}^{\prime} / \tau_{\varepsilon}^{P}+\tau_{\varepsilon} \rho_{I} \tau \beta_{3}^{2} \beta_{3}^{\prime} / \tau_{\varepsilon}^{P}\right)+\tau \rho_{I} \tau_{\varepsilon}\left(1+\beta_{3}\right) \beta_{3}^{2} / \tau_{\varepsilon}^{P^{2}}>0 \\
\Longleftrightarrow-\left(1+\beta_{3}\right)^{2} \beta_{3}^{\prime}-\tau 2 \beta_{3} \beta_{3}^{\prime} / \tau_{\varepsilon}^{P}-\tau \beta_{3}^{2} \beta_{3}^{\prime} / \tau_{\varepsilon}^{P}+\tau\left(1+\beta_{3}\right) \beta_{3}^{2} / \tau_{\varepsilon}^{P^{2}}>0 \\
\Longleftrightarrow \frac{r\left(-(1+r)\left(\tau_{\theta}+(\mu a)^{2} \tau_{u}\right)\left(\tau_{\varepsilon}^{P}+\tau_{\theta}+(\mu a)^{2} \tau_{u}\right)^{2}+r \tau_{\varepsilon}\left(\tau_{\varepsilon}^{P^{2}}(1+r)-\left(t+(\mu a)^{2} \tau_{u}\right)^{2}\right)\right)}{\left(\tau_{\varepsilon}^{P}+\tau_{\theta}+(\mu a)^{2} \tau_{u}\right)^{4}}>0,
\end{array}
$$

which holds for $r \rightarrow \infty$, since the positive term grows at a cubic rate while the negative terms grow at most at a quadratic rate. Thus, for $r>0$ large enough, $\operatorname{RHS}_{\tau_{\varepsilon}^{P}}>0$, hence $\partial a / \partial \tau_{\varepsilon}^{P}>0$.

Price informativeness (15) increases in $|a|, \mu, \tau_{u}$, decreases in $\tau_{\theta}, \tau_{\varepsilon}^{P}$, and does not change with $\psi_{4} / \psi_{3}, \tau_{\varepsilon}, \rho_{I}$. The total effect of parameter $t$ on price informativeness is

$$
\frac{d \mathrm{PI}}{d t}=\frac{\partial \mathrm{PI}}{\partial t}+\frac{\partial \mathrm{PI}}{\partial|a|} \cdot \frac{\partial|a|}{\partial t}
$$

Thus, all of the above comparative statics regarding $a$ also apply to price informativeness except for $\tau_{\theta}$ and $\tau_{\varepsilon}^{P}$, which decrease price informativeness directly $\left(\partial \mathrm{PI} / \partial \tau_{\theta}<0\right)$ but increase $|a|\left(\partial \mathrm{PI} / \partial|a| \cdot \partial|a| / \partial \tau_{\theta}>0\right)$, making the effect potentially ambiguous. The effects of $\psi_{4} / \psi_{3}, \tau_{\varepsilon}, \rho_{I}$ are indirect via $a(\partial \mathrm{PI} / \partial t=0)$.

The indirect effect is very small for $|r| \rightarrow \infty$, which leads to $\mathrm{RHS}_{\tau_{\theta}} \rightarrow 0$ and $\mathrm{RHS}_{a} \rightarrow 0$, hence $\partial|a| / \partial \tau_{\theta}=\mathrm{RHS}_{\tau_{\theta}} /\left(\mathrm{RHS}_{a}-1\right) \rightarrow 0$. The same applies to $\tau_{\varepsilon}^{P}$. Consequently, for $\tau_{\theta}$ and $\tau_{\varepsilon}^{P}$, the direct effect on price informativeness dominates for large $|r|$, and price informativeness decreases with larger $\tau_{\theta}$ and $\tau_{\varepsilon}^{P}$.

ii. $\mathrm{RHS}_{r}<0$ for $r<0$ sufficiently negative. This follows from the fact that the last term in $\mathrm{RHS}_{r}$ (see (25)) is positive for $r<<0$ sufficiently negative due to the quadratic terms. Thus, $a<0$ decreases (and $|a|$ increases) in response to a less negative $\psi_{4} / \psi_{3}<0$.

$\mathrm{RHS}_{\mu}<0$, since the last term in (26) is positive for $r \rightarrow-\infty$. $\mathrm{RHS}_{\rho_{I}}>0$, since $\operatorname{RHS}(a)<0$ in equilibrium (Proposition 5). $\operatorname{RHS}_{\tau_{\theta}}<0$, since the last term in (27) is positive for $r \rightarrow-\infty$. $\mathrm{RHS}_{\tau_{\varepsilon}}<0$, since $1+\beta_{3}<0$ for $r<<0$, which makes the slope in (29) negative. It can similarly be verified that $\mathrm{RHS}_{\tau_{u}}<0$ and $\mathrm{RHS}_{\tau_{\varepsilon}^{P}}<0$ if $r<<0$. Since $r<<0$ implies $a<0$ in equilibrium (Proposition 5), a decrease in $a$ increases $|a|$. Consequently, the same arguments as in $(i$.) show that the same comparative statics results apply to price informativeness.

iii. Note that equilibrium uniqueness (i.e., a unique solution to (14)) implies $\operatorname{RHS}_{a}<1$, since $\operatorname{RHS}(a)$ converges to a positive constant as $a \rightarrow \infty$, hence the continuous $\operatorname{RHS}(a)$ 
must cross the 45-degree line from above in the unique intersection. Thus, for $r:=$ $\psi_{4} / \psi_{3}$, as in parts $(i$.) and (ii.), the implicit function theorem implies that $a$ increases if and only if $\mathrm{RHS}_{r}>0$ and decreases if and only if $\mathrm{RHS}_{r}<0$.

$\mathrm{RHS}_{r}$ is given in (25). Clearly, for any $r \geq 0$ it is negative, hence $a$ decreases with larger $r$. Since the unique solution is $a>0$ for $r \geq 0$ (Corollary 4), larger $r$ for $r \geq 0$ decreases $|a|$ and reduces price informativeness (15).

Moreover, $\mathrm{RHS}_{r}$ is negative for $r<0$ close enough to zero, since the last term evaluated at $r=0$ is positive and continuous in $r$. Hence $a$ decreases with larger $r$ in this case as well. Since $\operatorname{RHS}(a=0)>0$ for $r<0$ close to zero, the unique solution of (14) is $a>0$, thus larger $r$ decreases $|a|$ and price informativeness.

Finally, if $r<<0$ sufficiently negative, then $\mathrm{RHS}_{r}$ in (25) is negative, hence $a$ decreases in response to a larger $r$. Since $a<0$ if $r<<0$ (Proposition 5), a larger $r$ increases $|a|$ and price informativeness.

\section{Proof of Proposition 7.}

$i$. The equilibrium object $a$ is implicitly defined in (14). Clearly, as $\left|\psi_{4} / \psi_{3}\right| \rightarrow \infty$, the right hand side tends to zero for any value of $a$, hence the solution $|a| \rightarrow 0$. With transparency, the equilibrium trading aggressiveness is $a=\tau_{\varepsilon} / \rho_{I}>0$, since informed demand is

$$
\begin{array}{r}
X_{I}\left(s_{j}, p, s_{p}\right)=\frac{\mathbb{E}\left[\theta+i\left(s_{p}, p\right)-p \mid s_{j}, p, s_{p}\right]}{\rho_{I} \operatorname{Var}\left(\theta+i\left(s_{p}, p\right)-p \mid s_{j}, p, s_{p}\right)}=\frac{\mathbb{E}\left[\theta \mid s_{j}, p, s_{p}\right]+i\left(s_{p}, p\right)-p}{\rho_{I} \operatorname{Var}\left(\theta \mid s_{j}, p, s_{p}\right)} \\
=\frac{\frac{\tau_{\theta} \bar{\theta}+\tau_{\varepsilon} s_{j}+\tau_{\varepsilon} s_{p}+(\mu a)^{2} \tau_{u}\left(\frac{p-\gamma \tilde{b}}{\lambda \mu a}\right)}{\tau_{\theta}+2 \tau_{\varepsilon}+(\mu a)^{2} \tau_{u}}+i\left(s_{p}, p\right)-p}{\rho_{I} \frac{1}{\tau_{\theta}+2 \tau_{\varepsilon}+(\mu a)^{2} \tau_{u}}}
\end{array}
$$

and matching the coefficient on $s_{j}$ gives the result $a=\tau_{\varepsilon} / \rho_{I}>0$, which is larger for sufficiently large $\left|\psi_{4} / \psi_{3}\right|$. Since price informativeness (15) increases in $|a|$, the result also applies to informativeness.

ii. First, note that the solution to (14) for $r:=\psi_{4} / \psi_{3}=0$ is $a=\tau_{\varepsilon} / \rho_{I}$. Second, the derivative of the right hand side (RHS) of (14) in $r$, (25), equals $-\tau_{\varepsilon}^{2} /\left(\rho_{I} \tau\right)<0$ at $r=0$. Hence, if $\mathrm{RHS}_{a}$ is less than 1 (i.e., the right hand side of (14) crosses from above), then the implicit function theorem implies that $a>0$ decreases as $\psi_{4} / \psi_{3}$ marginally increases (see (24)), or put differently, $a>0$ increases as $\psi_{4} / \psi_{3}$ decreases from $\psi_{4} / \psi_{3}=0$, i.e., is negative and sufficiently small. Thus, due to continuity, transparency increases the trading aggressiveness for $\psi_{4} / \psi_{3}<0$ sufficiently close to zero.

It remains to show that if there exists an equilibrium, then there is a solution to (14) with $\mathrm{RHS}_{a}$ less than 1. The right hand side of (14) is positive in the neighborhood of $a=0$ and $\psi_{4} / \psi_{3}=0$, and the RHS converges to $\tau_{\varepsilon}$ as $a \rightarrow \infty$ for any finite $\psi_{4} / \psi_{3}$. 
Hence, the RHS has to cross the LHS $y=a$ of (14) at least once from above, so that $\mathrm{RHS}_{a}<1$ at this solution.

\section{References}

Berg, J., F. Nelson, And T. Rietz (2008): "Prediction market accuracy in the long run," International Journal of Forecasting, 24, 285-300.

Berger, A. N., S. M. Davies, and M. J. Flannery (2000): "Comparing Market and Supervisory Assessments of Bank Performance: Who Knows What When?" Journal of Money, Credit \& Banking, 32, 641-641.

Bernanke, B. S. And M. Woodford (1997): "Inflation forecasts and monetary policy," Journal of Money, Credit, and Banking, 29, 653-684.

Birchler, U. W. And M. Facchinetti (2007): "Self-destroying prophecies? The endogeneity pitfall in using market signals as triggers for prompt corrective action," Working paper.

Blinder, A. S., M. Ehrmann, M. Fratzscher, J. De Haan, And D.-J. Jansen (2008): "Central Bank Communication and Monetary Policy: A Survey of Theory and Evidence," Journal of Economic Literature, 46, 910-945.

Bond, P. And I. Goldstein (2015): "Government intervention and information aggregation by prices," The Journal of Finance, 70, 2777-2812.

Bond, P., I. Goldstein, And E. S. Prescott (2010): "Market-based corrective actions," Review of Financial Studies, 23, 781-820.

Breon-Drish, B. (2015): "On Existence and Uniqueness of Equilibrium in a Class of Noisy Rational Expectations Models," Review of Economic Studies.

Cowgill, B. And E. Zitzewitz (2015): "Corporate Prediction Markets: Evidence from Google, Ford, and Firm X," Review of Economic Studies, rdv014.

Daniel, K., D. Hirshleifer, and A. Subrahmanyam (1998): "Investor psychology and security market under-and overreactions," The Journal of Finance, 53, 1839-1885.

Dow, J., I. Goldstein, And A. Guembel (2017): "Incentives for information production in markets where prices affect real investment," Journal of the European Economic Association, Forthcoming. 
Dreber, A., T. Pfeiffer, J. Almenberg, S. Isaksson, B. Wilson, Y. Chen, B. A. Nosek, And M. Johannesson (2015): "Using prediction markets to estimate the reproducibility of scientific research," Proceedings of the National Academy of Sciences, $112,15343-15347$.

Edmans, A., I. Goldstein, And W. Jiang (2015): "Feedback Effects, Asymmetric Trading, and the Limits to Arbitrage," American Economic Review, 105, 3766-97.

FinAnCial Times (2015): "EU ombudsman rebukes ECB over market leaks," https: //www.ft.com/content/a2af6b10-0532-11e5-9627-00144feabdc0.

Foucault, T. And T. Gehrig (2008): "Stock price informativeness, cross-listings, and investment decisions," Journal of Financial Economics, 88, 146-168.

GeraAts, P. M. (2002): "Central bank transparency," The Economic Journal, 112, F532F565.

Goldstein, I. And A. Guembel (2008): "Manipulation and the allocational role of prices," Review of Economic Studies, 75, 133-164.

Goldstein, I., E. Ozdenoren, And K. Yuan (2013): "Trading frenzies and their impact on real investment," Journal of Financial Economics, 109, 566-582.

Gropp, R., J. Vesala, And G. Vulpes (2006): "Equity and bond market signals as leading indicators of bank fragility," Journal of Money, Credit, and Banking, 38, 399-428.

Grossman, S. J. And J. E. Stiglitz (1980): "On the impossibility of informationally efficient markets," American Economic Review, 70, 393-408.

Hellwig, M. F. (1980): "On the aggregation of information in competitive markets," Journal of Economic Theory, 22, 477-498.

Kim, D. H., D. R. Osborn, And M. Sensier (2005): "Nonlinearity in the Fed's monetary policy rule," Journal of Applied Econometrics, 20, 621-639.

Ozdenoren, E. And K. YUAn (2008): "Feedback effects and asset prices," The Journal of Finance, 63, 1939-1975.

PÁlvÖlgyi, D. AND G. VEnter (2015): "Multiple equilibria in noisy rational expectations economies," Working Paper.

Prescott, E. S. (2012): "Contingent capital: the trigger problem," Federal Reserve Bank of Richmond Economic Quarterly, 98, 33-50.

RADNER, R. (1979): "Rational expectations equilibrium: Generic existence and the information revealed by prices," Econometrica, 655-678. 
Ravn, S. (2012): "Has the Fed Reacted Asymmetrically to Stock Prices?" The BE Journal of Macroeconomics, 12.

REIs, R. (2013): "Central bank design," The Journal of Economic Perspectives, 27, 17-43.

$\begin{array}{cccccc}\text { REUTERS (2008): } & \text { "AIG } & \text { gets } \$ 150 \text { billion government bailout; } \\ \text { posts } & \text { huge } & \text { loss," } & \text { https://www.reuters.com/article/us-aig/ } \\ \text { aig-gets-150-billion-government-bailout-posts-huge-loss-idUSTRE4A92FM20081110. }\end{array}$

Siemroth, C. (2017): "When can decision makers learn from financial market prices?" Working paper.

Sun, Y. (2006): "The exact law of large numbers via Fubini extension and characterization of insurable risks," Journal of Economic Theory, 126, 31-69.

Sundaresan, S. AND Z. WANG (2015): "On the design of contingent capital with a market trigger," Journal of Finance, 70, 881-920.

Surico, P. (2007): "The Fed's monetary policy rule and US inflation: The case of asymmetric preferences," Journal of Economic Dynamics and Control, 31, 305-324.

VIVES, X. (2010): Information and learning in markets: The impact of market microstructure, Princeton University Press.

WeISE, C. L. (1999): "The asymmetric effects of monetary policy: A nonlinear vector autoregression approach," Journal of Money, Credit and Banking, 85-108.

Woodford, M. (1994): "Nonstandard Indicators for Monetary Policy: Can Their Usefulness Be Judged from Forecasting Regressions?" in Monetary Policy, ed. by N. G. Mankiw, University of Chicago Press. 